\title{
Y-12 GROUNDWATER PROTECTION PROGRAM GROUNDWATER AND SURFACE WATER SAMPLING AND ANALYSIS PLAN FOR CALENDAR YEAR 2014
}

September 2013

\section{NATIONAL SECURITY COMPLEX}

for the

Environmental Compliance Department Environment, Safety, and Health Division Y-12 National Security Complex Oak Ridge, Tennessee 37831

Managed by

Babcock \& Wilcox Technical Services Y-12, LLC for the U.S. DEPARTMENT OF ENERGY under contract No. DE-AC05-00OR22800 


\section{DISCLAIMER}

This report was prepared as an account of work sponsored by an agency of the United States Government. Neither the United States Government nor any agency thereof, nor any of their employees, makes any warranty, express or implied, or assumes any legal liability or responsibility for the accuracy, completeness, or usefulness of any information, apparatus, product, or process disclosed, or represents that its use would not infringe privately owned rights. Reference herein to any specific commercial product, process, or service by trade name, trademark, manufacturer, or otherwise, does not necessarily constitute or imply its endorsement, recommendation, or favoring by the United States Government or any agency thereof. The views and opinions of authors expressed herein do not necessarily state or reflect those of the United States Government or any agency thereof. 


\title{
Y-12 GROUNDWATER PROTECTION PROGRAM GROUNDWATER AND SURFACE WATER \\ SAMPLING AND ANALYSIS PLAN \\ FOR CALENDAR YEAR 2014
}

September 2013

\author{
Prepared by \\ Elvado Environmental LLC \\ Under Subcontract No. 4300087609
}

for the

\begin{abstract}
Environmental Compliance Department Environment, Safety, and Health Division Y-12 National Security Complex

Oak Ridge, Tennessee 37831
\end{abstract}

\section{Managed by}

Babcock \& Wilcox Technical Services Y-12, LLC for the U.S. DEPARTMENT OF ENERGY under contract No. DE-AC05-00OR22800 


\section{TABLE OF CONTENTS}

$\underline{\text { Section }} \quad \underline{\text { Page }}$

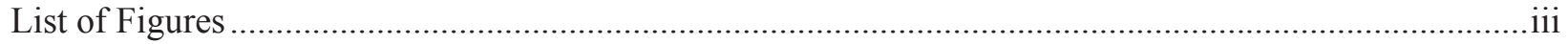

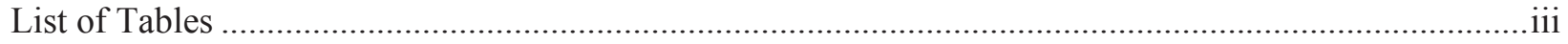

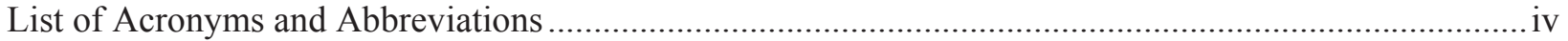

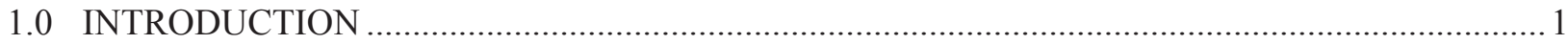

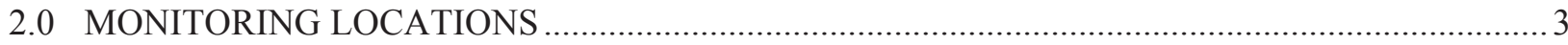

3.0 FIELD MEASUREMENTS AND ANALYTICAL PARAMETERS ..............................................5

4.0 SAMPLE PLANNING, COLLECTION, AND HANDLING ..................................................... 7

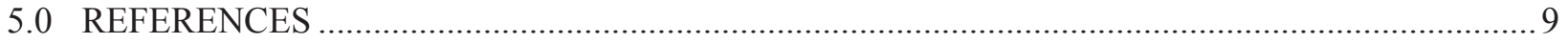

\section{APPENDICES:}

$\begin{array}{ll}\text { A } & \text { FIGURES } \\ \text { B } & \text { TABLES } \\ \text { C } & \text { CY 2014 GROUNDWATER MONITORING SCHEDULES } \\ \text { D } & \text { ADDENDA TO THE CY 2014 SAMPLING AND ANALYSIS PLAN } \\ \text { E } & \text { LABORATORY REQUIREMENTS } \\ & \text { (Bottle Lists, Holding Times, Turnaround Times, Elevated Minimum Detectable Activity) } \\ \text { F } & \text { MANAGEMENT OF PURGED GROUNDWATER } \\ & \text { F.1 WASTE MANAGEMENT PLAN } \\ & \text { F.2 WASTEWATER STREAM IDENTIFICATION FOR PURGED GROUNDWATER }\end{array}$




\section{List of Figures}

Figure

$\underline{\text { Page }}$

A.1 Hydrogeologic regimes at the Y-12 National Security Complex ............................................A-1

A.2 CY 2014 sampling locations in the Bear Creek Hydrogeologic Regime................................... A-2

A.3 CY 2014 sampling locations in the Chestnut Ridge Hydrogeologic Regime ............................ A-3

A.4 CY 2014 sampling locations in the Upper East Fork Poplar Creek

Hydrogeologic Regime

A.5 CY 2014 surface water sampling locations north of Pine Ridge

A.6 Westbay ${ }^{\mathrm{TM}}$ monitoring system sampling port depths in well GW-726.................................. A-6

A.7 Westbay ${ }^{\mathrm{TM}}$ monitoring system sampling port depths in well GW-722 ................................ A-7

A.8 Westbay ${ }^{\mathrm{TM}}$ monitoring system sampling port depths in well GW-934................................... A-8

\section{List of Tables}

Table

B.1 Sampling locations, frequency, and analytical parameters for groundwater and surface water monitoring during CY 2014

B.2 Field measurements and analytes that comprise the elementary parameter groups for CY 2014 groundwater and surface water samples 


\section{List of Acronyms and Abbreviations}

$\mathrm{ACO}$

$\mathrm{B} \& \mathrm{~W} \mathrm{Y}-12$

Bear Creek Regime

Chestnut Ridge Regime

CY

DOE

East Fork Regime

GWPP

GWMS

redox

SPM

VOCs

$\mathrm{Y}-12$
Analytical Chemistry Organization

Babcock \& Wilcox Technical Services Y-12, LLC

Bear Creek Hydrogeologic Regime

Chestnut Ridge Hydrogeologic Regime

calendar year

U.S. Department of Energy

Upper East Fork Poplar Creek Hydrogeologic Regime

Groundwater Protection Program

Groundwater Monitoring Schedule

oxidation-reduction potential

selective parameter monitoring

volatile organic compounds

Y-12 National Security Complex 


\subsection{INTRODUCTION}

This plan provides a description of the groundwater and surface water quality monitoring activities planned for calendar year (CY) 2014 at the U.S. Department of Energy (DOE) Y-12 National Security Complex (Y-12) that will be managed by the Y-12 Groundwater Protection Program (GWPP). Groundwater and surface water monitoring is performed by the GWPP during CY 2014 to achieve the following goals:

- to protect the worker, the public, and the environment;

- to maintain surveillance of existing and potential groundwater contamination sources;

- to provide for the early detection of groundwater contamination and determine the quality of groundwater and surface water where contaminants are most likely to migrate beyond the Oak Ridge Reservation property line;

- to identify and characterize long-term trends in groundwater quality at Y-12; and

- to provide data to support decisions concerning the management and protection of groundwater resources.

Groundwater and surface water monitoring will be performed in three hydrogeologic regimes at Y-12: the Bear Creek Hydrogeologic Regime (Bear Creek Regime), the Upper East Fork Poplar Creek Hydrogeologic Regime (East Fork Regime), and the Chestnut Ridge Hydrogeologic Regime (Chestnut Ridge Regime). The Bear Creek and East Fork regimes are located in Bear Creek Valley and the Chestnut Ridge Regime is located south of Y-12 (Figure A.1). Additional surface water monitoring will be performed north of Pine Ridge along the boundary of the Oak Ridge Reservation.

The following sections of this report provide details regarding the CY 2014 groundwater and surface water monitoring activities. Section 2 describes the monitoring locations in each regime and the processes used to select the sampling locations. A description of the field measurements and laboratory analytes is provided in Section 3. Sample collection methods and procedures are described in Section 4, and Section 5 lists the documents cited for more detailed operational and technical information.

The narrative sections of the report reference several appendices. Figures (maps and diagrams) and tables (excluding a data summary table presented in Section 4) are in Appendix A and Appendix B, respectively. Groundwater Monitoring Schedules (when issued throughout CY 2014) will be inserted in Appendix C, and addenda to this plan (if issued) will be inserted in Appendix D. Laboratory requirements (bottle lists, holding times, etc.) are provided in Appendix E, and an approved Waste Management Plan is provided in Appendix F.

Modifications to the CY 2014 monitoring program may be necessary during implementation. Changes in programmatic requirements may alter the analytes specified for selected monitoring wells or may add or remove wells from the planned monitoring network. Each modification to the monitoring program will be approved by the Y-12 GWPP manager and documented as an addendum to this sampling and analysis plan. 


\subsection{MONITORING LOCATIONS}

The Y-12 GWPP monitoring network for CY 2014 includes 115 monitoring locations (Table B.1): 40 located in the Bear Creek Regime (Figure A.2), nine located in the Chestnut Ridge Regime (Figure A.3), 63 located in the East Fork Regime (Figure A.4), and three located north of Pine Ridge (Figure A.5). These monitoring locations were selected based on the sampling frequencies defined in the Y-12 GWPP monitoring optimization plan (Babcock \& Wilcox Technical Services [B\&W] Y-12, LLC 2013a). The sampling frequencies for monitoring locations in CY 2014 include semiannual, annual, biennial (even-numbered CYs), and pentennial (every five years) monitoring.

Groundwater samples will be collected from a total of 101 monitoring wells, including 34 wells in the Bear Creek Regime (Figure A.2), four wells in the Chestnut Ridge Regime (Figure A.3), and 63 wells in the East Fork Regime (Figure A.4). One well in the Bear Creek Regime (GW-726) and two wells in the East Fork Regime (GW-722 and GW-934) contain a Westbay ${ }^{\mathrm{TM}}$ multiport sampling system that allows collection of groundwater samples from several discrete depth intervals (Table B.1). Well GW-726 will have samples collected from seven ports (Figure A.6). Well GW-722 will have samples collected from five ports (Figure A.7) and well GW-934 will have samples collected from eight ports (Figure A.8).

Samples of groundwater discharging from four natural springs will be collected. Springs SS-4 and SS-5 are located in the Bear Creek Regime (Figure A.2), and springs SCR2.1SP and SCR2.2SP are in the Chestnut Ridge Regime (Figure A.3).

The Y-12 GWPP collects surface water samples from selected locations because of the potential interaction between groundwater and surface water (e.g., gaining and losing reaches of creeks that reflect groundwater discharge and recharge, respectively). During CY 2014, surface water samples will be collected from a total of ten sampling locations, including four locations in the Bear Creek Regime, three locations in the Chestnut Ridge Regime, and three locations north of Pine Ridge. In the Bear Creek Regime, samples will be collected from three stations (BCK-04.55, BCK-09.40, and BCK-11.97) in the main channel of Bear Creek, and from one station (NT-01) in a northern tributary to Bear Creek (Figure A.2). The tributaries located in the Chestnut Ridge Regime have been numbered from west to east (SCR1 through SCR5) and surface water samples will be collected from three of the tributaries at stations (SCR1.5SW, SCR3.5SW, and S17 [located in SCR5]) located along the north side of Bethel Valley Road (Figure A.3). The surface water sampling locations north of Pine Ridge (Figure A.5) include a tributary near the Scarboro Community (NPR07.0SW), a tributary to Mill Branch (NPR23.0SW), and Gum Hollow Branch near Country Club Estates (GHK2.51ESW). 


\subsection{FIELD MEASUREMENTS AND ANALYTICAL PARAMETERS}

Before collecting samples at most monitoring locations, field personnel will record applicable field measurements (Table B.2), including:

- depth to the static water level in monitoring wells;

- $\mathrm{pH}$;

- water temperature;

- conductivity;

- dissolved oxygen; and

- oxidation-reduction potential (redox)

Field measurement of dissolved oxygen and redox will not be obtained for sampling ports of monitoring wells equipped with a Westbay ${ }^{\mathrm{TM}}$ multiport sampling system. Also, the potentiometric head (in $\mathrm{ft}$ ) will be calculated from subsurface pressure measurements for each Westbay ${ }^{\mathrm{TM}}$ sampling zone instead of measuring the depth to the static water level.

For this Sampling and Analysis Plan, specific analytes are grouped by analytical method or by type (e.g., trace metals) and referenced as elementary parameter groups (Table B.1 and Table B.2). In addition to field measurements, most of the groundwater samples and all surface water samples will be analyzed for the following suite of parameters (identified as the Standard Administrative Parameter Group):

- total suspended solids and total dissolved solids;

- major anions;

- trace metals (includes major cations);

- a comprehensive suite of volatile organic compounds (VOCs); and

- gross alpha and gross beta activity.

Selective parameter monitoring (SPM) will be performed on samples from selected monitoring wells with analytical results for at least eight samples obtained since January 1991. Historical data must clearly demonstrate that the selected parameters are the contaminants of concern and provide sufficient data to characterize the other parameters without additional analyses. For example, samples from 33 monitoring wells will be analyzed only for VOCs (Table B.1), and historical data for these locations show consistently low results for inorganic and radiochemical analytes. The SPM elementary parameter groups reflect analytical methods (Table B.2) and are designed to obtain the data necessary to meet requirements of the GWPP monitoring program.

Samples from selected locations will be analyzed for specific radionuclides to supplement gross alpha and/or gross beta activity results. In cases where the gross activity reporting limits are elevated from interferences caused by a high dissolved solid content of the groundwater sample, the laboratory will request isotopic analyses (see Appendix E). 


\subsection{SAMPLE PLANNING, COLLECTION, AND HANDLING}

The monitoring locations to be sampled during CY 2014 are grouped by hydrogeologic regime to provide geographic areas for planning and tracking purposes. The CY quarter for sample collection at each monitoring location is provided in Table B.1.

A Groundwater Monitoring Schedule (GWMS) will be prepared by GWPP personnel for each quarterly sampling event of CY 2014. Each GWMS will be issued before sample collection begins, will specify the sequence for collecting samples from the monitoring locations scheduled, and will include information necessary for field personnel to collect the required samples (e.g., containment requirements and previous pumping rates used to sample each well). The GWMS is an integral part of this document, and when issued electronically, the GWMS for each CY 2014 sampling event is to be printed and inserted (Appendix C) by the recipient.

Unfiltered samples will be collected semiannually from four monitoring wells and once from the rest of the monitoring locations during CY 2014 (Table B.1). As summarized below, the number of samples to be collected during each CY quarter will range from 21 to 54, for an annual total of 137 samples.

\begin{tabular}{|c|c|c|c|c|}
\hline \multirow{2}{*}{$\begin{array}{c}\text { HYDROGEOLOGIC } \\
\text { REgIME/AREA }\end{array}$} & $\mathbf{4}$ NUMBER OF SAMPLES PER QUARTER OF CY 2014 \\
\cline { 2 - 5 } & $\mathbf{1 s t}$ & 2nd & 3rd & 4th \\
\hline Bear Creek Regime & 16 & 0 & 31 & 0 \\
Chestnut Ridge Regime & 0 & 9 & 0 & 0 \\
East Fork Regime & 23 & 12 & 20 & 23 \\
North of Pine Ridge & 0 & 0 & 3 & 0 \\
\hline Total: & $\mathbf{3 9}$ & $\mathbf{2 1}$ & $\mathbf{5 4}$ & $\mathbf{2 3}$ \\
\hline
\end{tabular}

Personnel from the Environmental Sampling Section of the Y-12 Environment Compliance Department will be responsible for collection, transportation, and chain-of-custody control of all groundwater and surface water samples. Based on the analytical parameters for the CY 2014 monitoring locations (Table B.1 and Table B.2), personnel with the Y-12 Analytical Chemistry Organization (ACO) prepare bottle lists that specify the sample container type, size, preservative, and the laboratory test identification needed for each sampling location (see Appendix E). Additionally, ACO personnel will generate a weekly tracking report to record the sample collection date and time for each monitoring location and the status of analyses. Sample collection will be performed in accordance with the most recent version of operating procedures for obtaining groundwater samples (B\&W Y-12 2011, B\&W Y-12 2012, B\&W Y-12 2013b, and B\&W Y-12 2013c) and surface water samples (B\&W Y-12 2010). All field and laboratory activities will be performed in accordance with applicable requirements of the Y-12 Integrated Safety Management System and task-specific job hazard analyses.

Groundwater samples will be collected using the low-flow minimal drawdown method (low-flow method) during CY 2014 from most of the monitoring wells (Table B.1). A passive (no purging) sampling method will be used to collect samples at three monitoring wells by collecting a sample using the dedicated pump without purging. Additionally, groundwater samples from three wells that are equipped with a Westbay ${ }^{\mathrm{TM}}$ multiport sampling system will be collected following applicable procedures.

For the low-flow method, a bladder pump is permanently installed in each well that is scheduled for sample collection. If well construction prevents permanent installation (e.g., flush-mounted wells), then the pump and tubing will be installed at least 24 hours before sample collection and will be removed when sampling is 
completed. In accordance with the groundwater sampling procedure for the low-flow method (B\&W Y-12 2012), groundwater is purged, and subsequently sampled, from the well at a flow rate $(<300$ milliliters per minute $[\mathrm{ml} / \mathrm{min}])$ which ensures minimal drawdown of the static water level, therefore isolating the stagnant water column above the intake of the pump. Groundwater samples are collected from a well after the water level is in steady-state drawdown $(<0.1 \mathrm{ft}$ over a 15 -minute interval $)$ and field parameters $(\mathrm{pH}$, conductivity, water temperature, redox, and dissolved oxygen) have stabilized (minimal variation over four consecutive readings).

A "no-purge method" may be used for wells with low-flow sampling histories that consistently demonstrate very low pumping rate $(<50 \mathrm{ml} / \mathrm{min})$ to meet the minimal drawdown requirement during purging and sample collection. During CY 2014, the no-purge method will be used to collect groundwater samples from wells GW-065 and GW-623 in the Bear Creek Regime and well GW-275 in the East Fork Regime (Table B.1). For this method, field measurements will be obtained and groundwater samples will be collected after pumping the stagnant water (calculated volume) from the tubing.

Groundwater sampling and pressure profiling using a Westbay ${ }^{\mathrm{TM}}$ multiport sampling system at wells GW-726 (Figure A.6), GW-722 (Figure A.7) and GW-934 (Figure A.8) will be performed in accordance with the applicable operating procedures (B\&W Y-12 2013b and B\&W Y-12 2013c). The groundwater samples from each sampling port will be collected in 250-milliliter nonvented stainless steel Westbay ${ }^{\mathrm{TM}}$ sample collection bottles filled at the designated depth in the well. Once filled, the bottles will be raised to the surface and the groundwater will be transferred to laboratory sample containers. The sample collection bottles will be lowered, filled, and retrieved as many times as needed to completely fill the laboratory sample bottles. Groundwater in the first sample collection bottles retrieved from each sampling port will be used as a "formation rinse" to obtain field measurements and to condition the sample collection bottle for each zone. To minimize cross contamination between monitored intervals in a well, groundwater samples will be collected in order from the least contaminated zone to most contaminated zone, based on historical data. Sample bottles will be rinsed with deionized water between zones to remove residual contamination.

In addition to the groundwater and surface water samples, field blanks and equipment rinsate samples will be collected at the frequencies and analyzed for VOCs (Table B.1). Field blank samples will be collected for at least $1 \%$ of the samples. Therefore, two field blank samples will be collected during CY 2014: one in the East Fork Regime during first quarter and one in the Bear Creek Regime during the third quarter. An equipment rinsate sample will be collected from each Westbay well immediately after field-cleaning the sampling equipment used to collect samples from the designated sampling port (Table B.1).

Trip blank samples and field duplicate samples will be prepared and handled in accordance with the Field Quality Control Samples operating procedure (B\&W Y-12 2009b) and will be analyzed using applicable procedures. Trip blank samples will be prepared for each cooler used to transport samples for VOC analyses. Because duplicate samples will be collected from at least $10 \%$ of the sampling locations, a total of 14 field duplicate samples will be collected during CY 2014: five in the Bear Creek Regime, one in the Chestnut Ridge Regime, and eight in the East Fork Regime (Table B.1).

All groundwater and surface water samples will be handled in accordance with procedures for chain-of-custody (B\&W Y-12 2009c) and relinquished to the appropriate Y-12 ACO laboratory that will perform the analyses. The Y-12 ACO laboratories will perform each analysis within established holding times and deliver results in hard copy and electronic format within established turnaround times (see Appendix E). 


\subsection{REFERENCES}

American Public Health Association. 1992. Standard Methods for Examination of Water and Wastewater, $18^{\text {th }}$ Edition. (referenced on Table B.2)

Babcock \& Wilcox Technical Services (B\&W) Y-12. 2009b. Field Quality Control Samples. B BW Y-12 Management Requirement prepared by the Environment, Safety, and Health Division (Y71-66-EC-003, Rev 09/16/09).

B\&W Y-12. 2009c. Sample Chain of Custody. B\&W Y-12 Management Requirement prepared by the Environment, Safety, and Health Division (Y71-66-EC-004, Rev 09/16/09).

B\&W Y-12. 2010. Liquid Grab Sampling. B\&W Y-12 Management Requirement prepared by the Environment, Safety, and Health Division (Y50-71-005, 10/19/10).

B\&W Y-12. 2011. Measurement of Static Water Level Elevation. B\&W Y-12 Management Requirement prepared by the Environment, Safety, and Health Division (Y50-71-015, Rev 1.1 06/07/11).

B\&W Y-12. 2012. Groundwater Sampling. B\&W Y-12 Management Requirement prepared by the Environment, Safety, and Health Organization (Y71-66-EC-116, 07/03/12).

B\&W Y-12. 2013a. Y-12 Groundwater Protection Program Monitoring Optimization Plan for Groundwater Monitoring Wells at the U.S. Department of Energy Y-12 National Security Complex, Oak Ridge, Tennessee. Prepared by Elvado Environmental LLC (Y/TS-2031/R2).

B\&W Y-12. 2013b. Pressure Profiling of Wells Equipped with Westbay ${ }^{\mathrm{TM}}$ Monitoring System Instrumentation. B\&W Y-12 Management Requirement prepared by the Environment, Safety, and Health Organization (Y71-66-EC-119).

B\&W Y-12. 2013c. Groundwater Sampling of Westbay ${ }^{\mathrm{TM}}$ Monitoring System Instrumented Wells. B\&W Y-12 Management Requirement prepared by the Environment, Safety, and Health Organization (Y71-66-EC-118).

Martin Marietta Energy Systems, Inc. 1990. Comprehensive Groundwater Monitoring Plan for the Oak Ridge Y-12 Plant. Prepared by Geraghty \& Miller, Inc. (Y/SUB/90-00206C/5) (Grid locations referenced on Table B.1)

U.S. Environmental Protection Agency. 1983. Methods for Chemical Analysis of Water and Wastes. (referenced on Table B.2)

U.S. Environmental Protection Agency. 1996. Test Methods for Evaluating Solid Waste Physical/Chemical Methods. (referenced on Table B.2) 
APPENDIX A

\section{FIGURES}




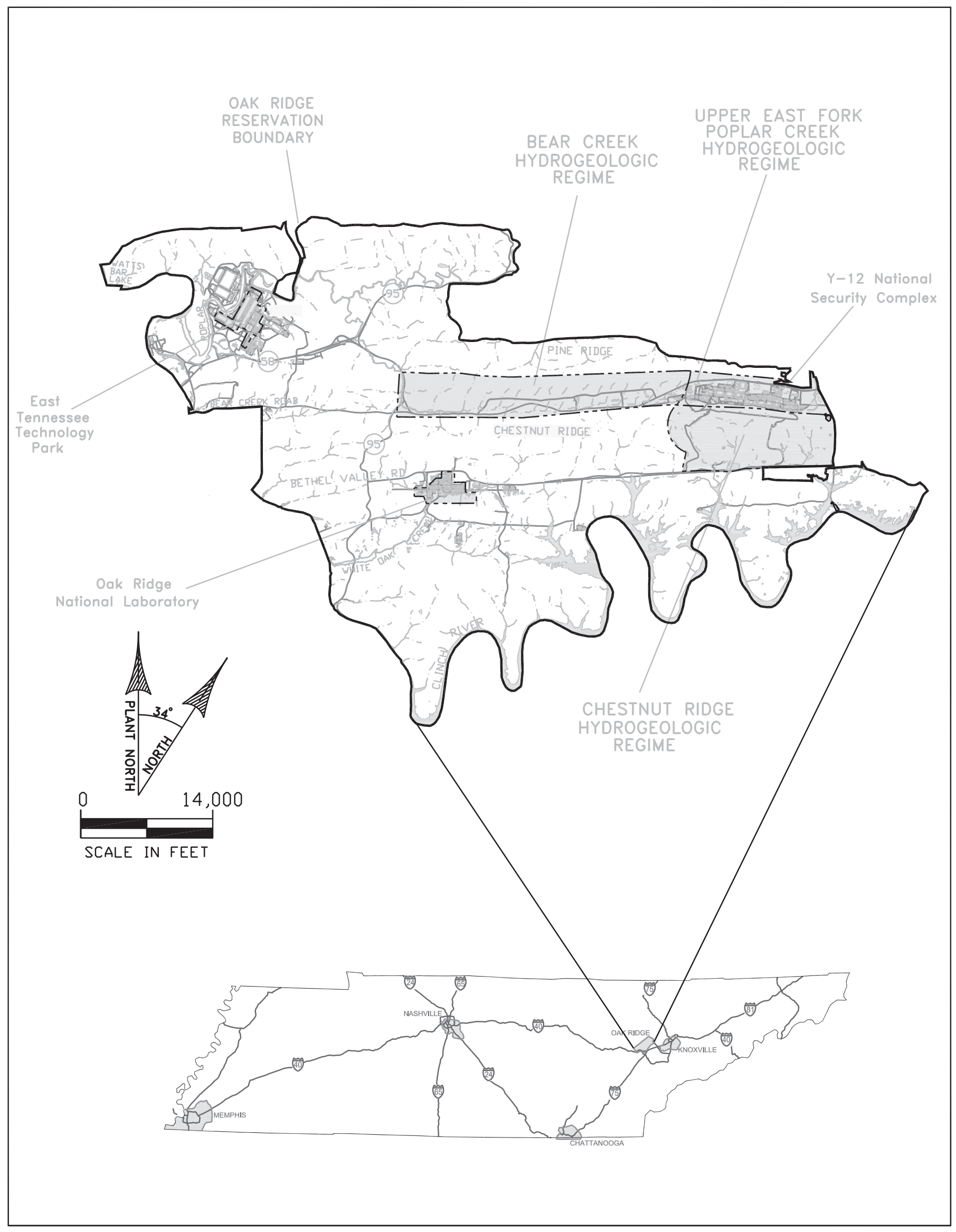

GWPP Fig1 09/23/08

Fig. A.1. Hydrogeologic regimes at the Y-12 National Security Complex. 


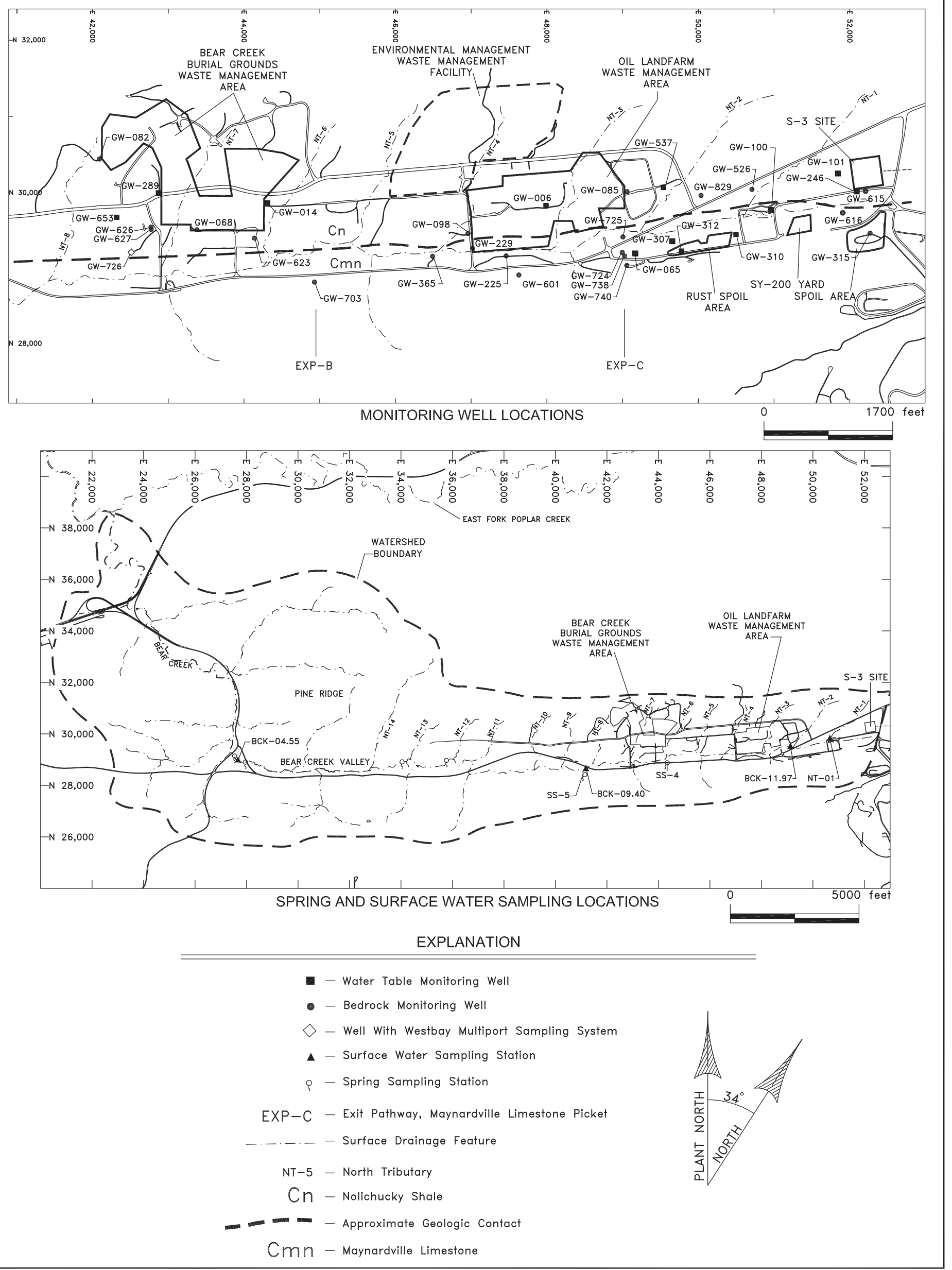




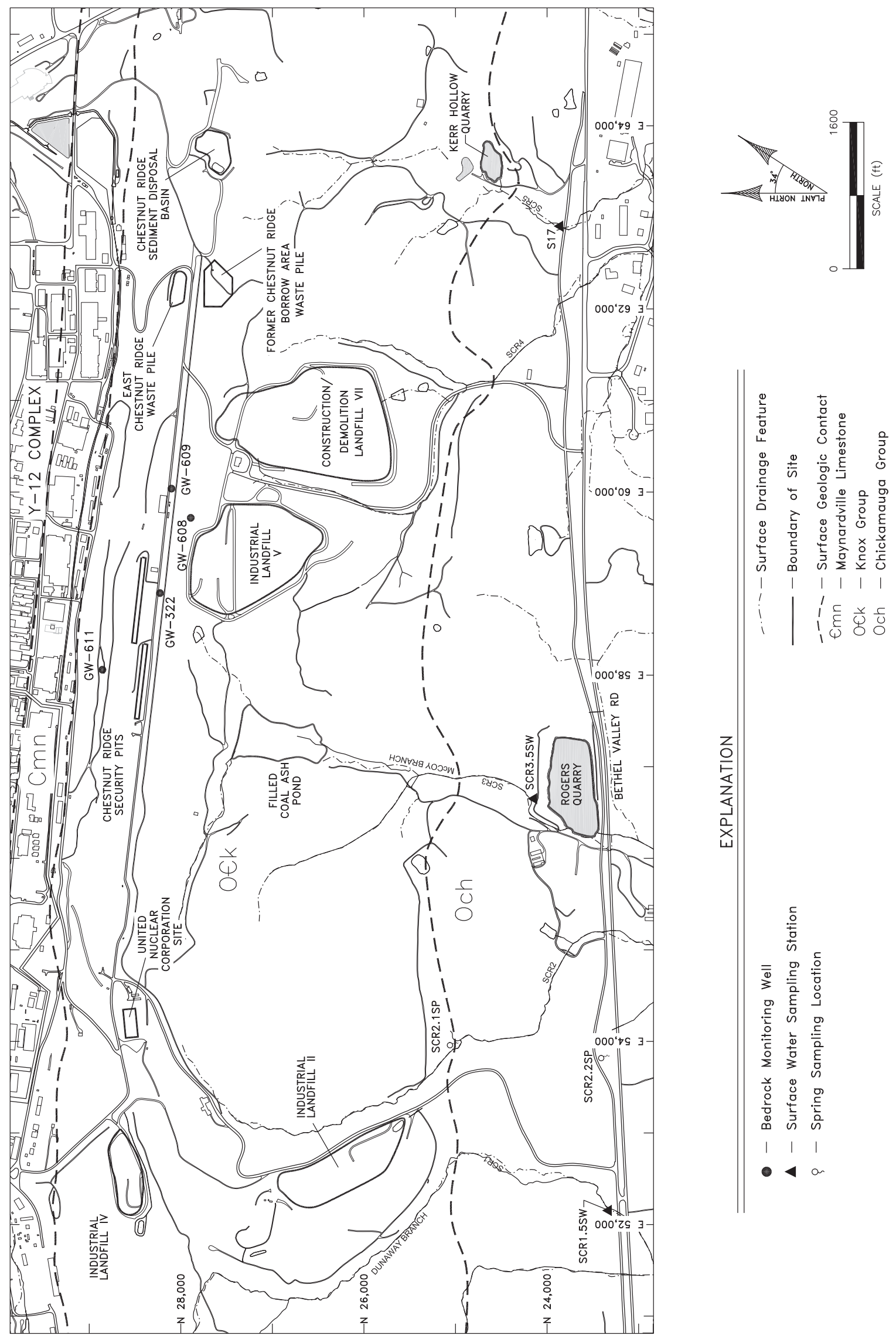

SAP14_3 09/23/13

Fig. A.3. CY 2014 sampling locations in the Chestnut Ridge Hydrogeologic Regime. 


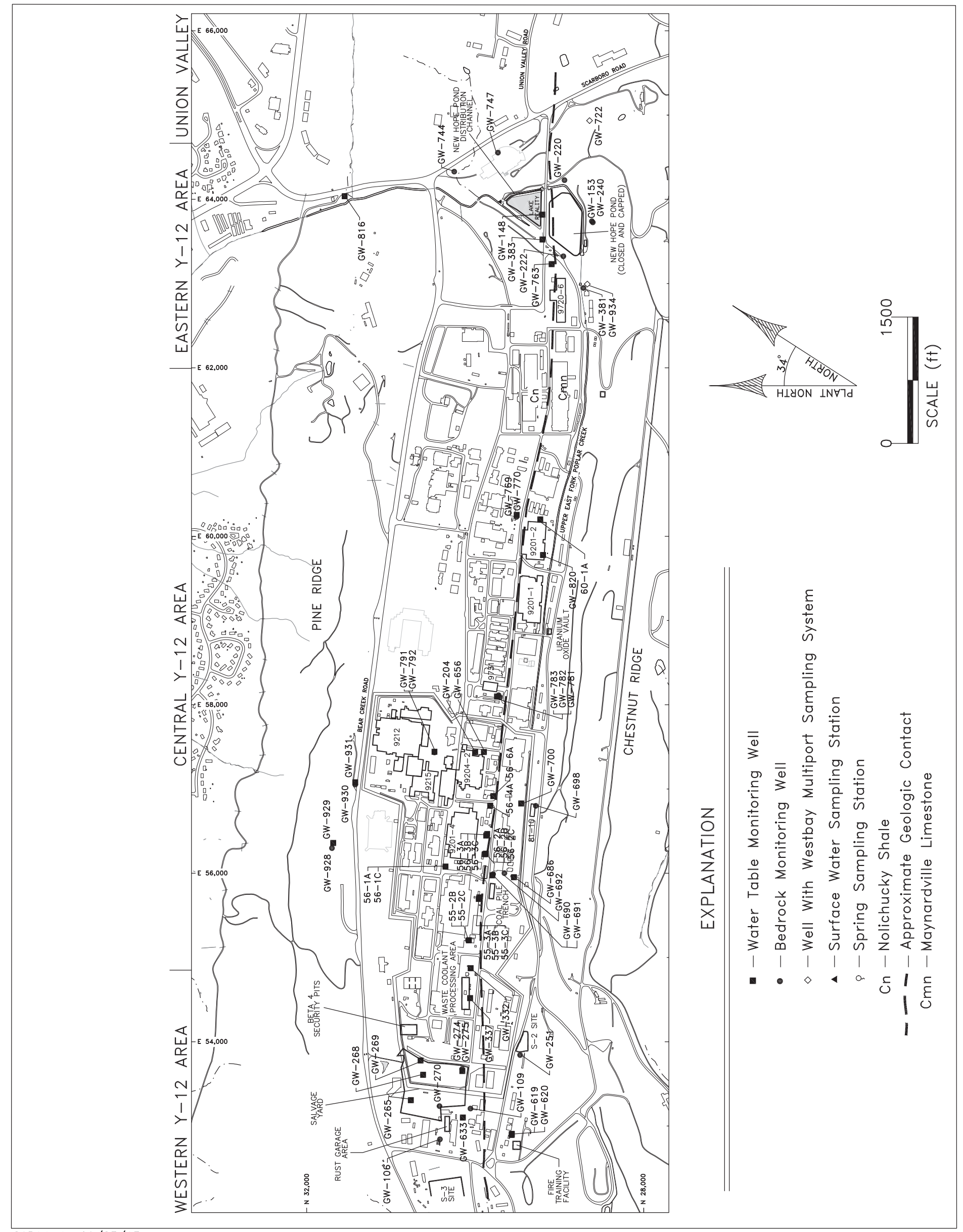

Fig. A.4. CY 2014 sampling locations in the Upper East Fork Poplar Creek Hydrogeologic Regime. 


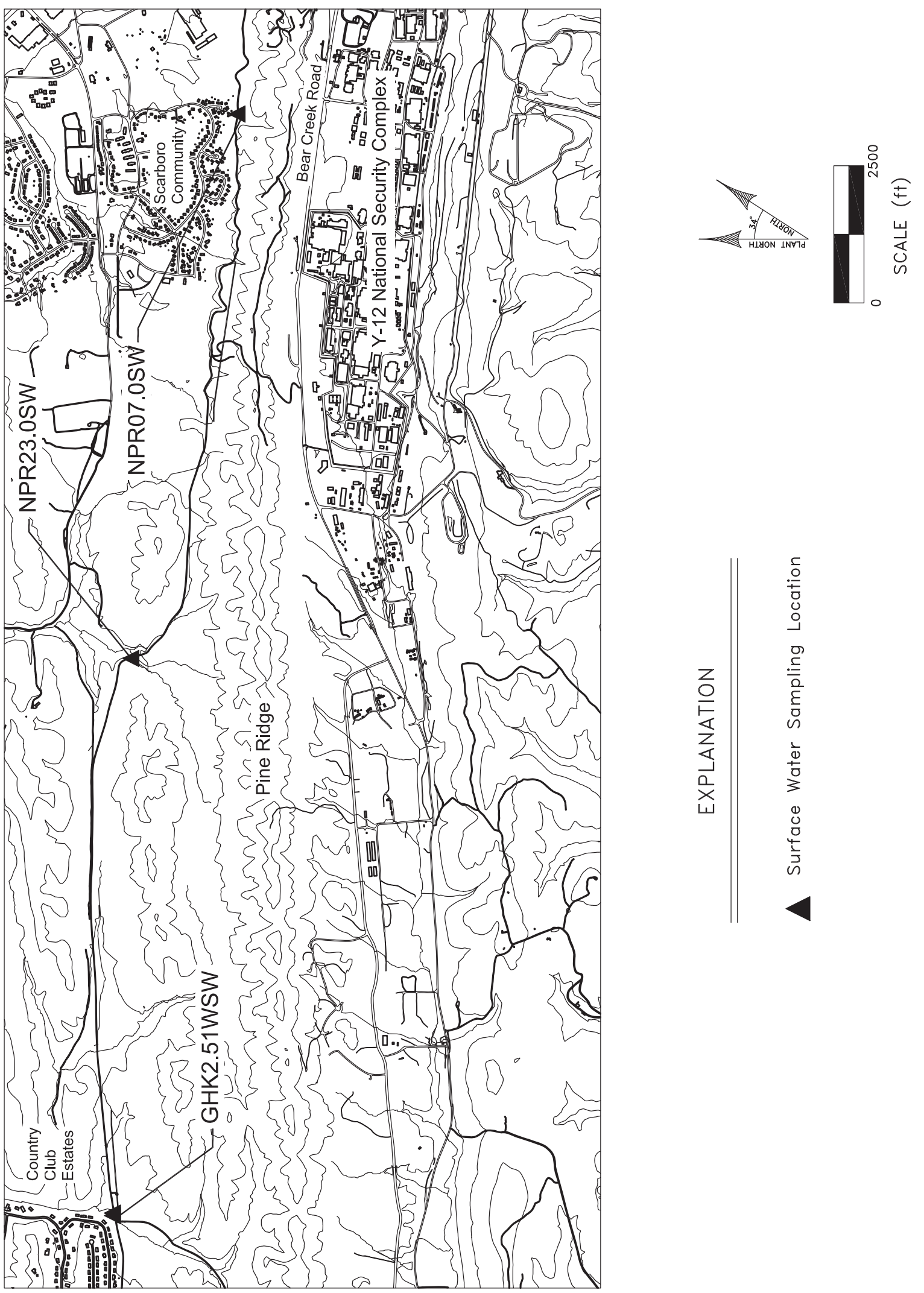




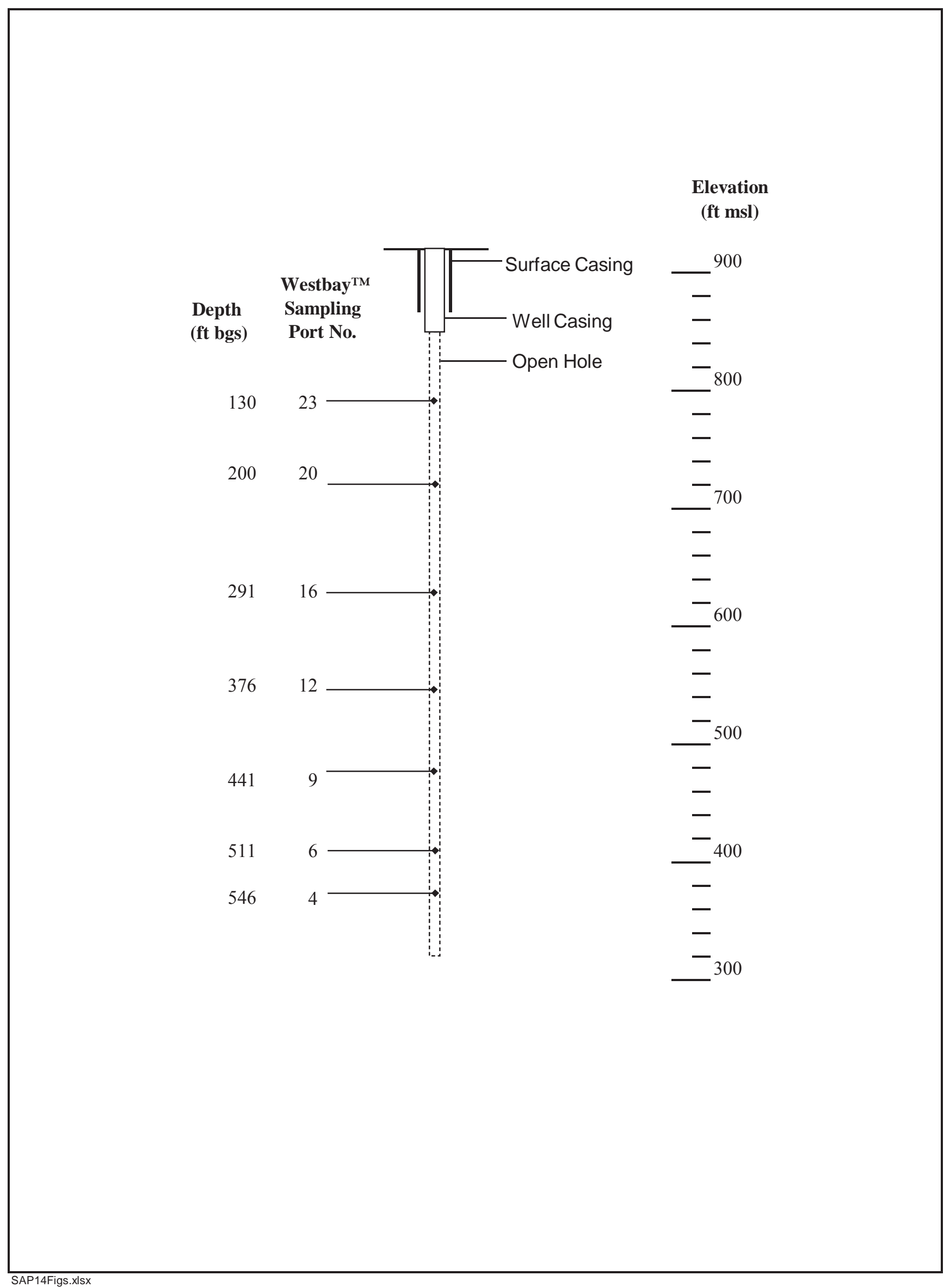

Fig. A.6. Westbay ${ }^{\mathrm{TM}}$ monitoring system sampling port depths in well GW-726.

A-6 


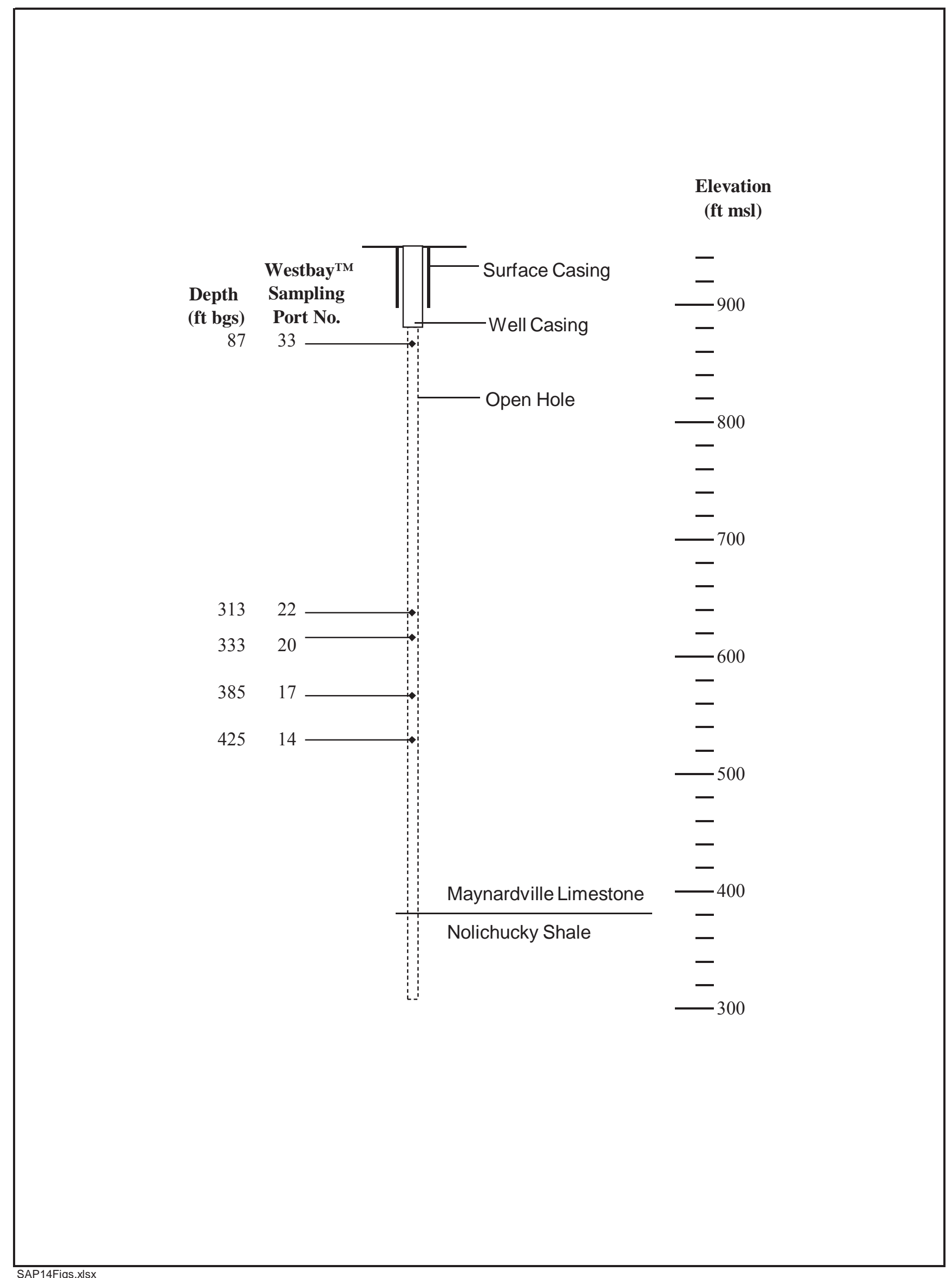

Fig. A.7. Westbay ${ }^{\mathrm{TM}}$ monitoring system sampling port depths in well GW-722. 


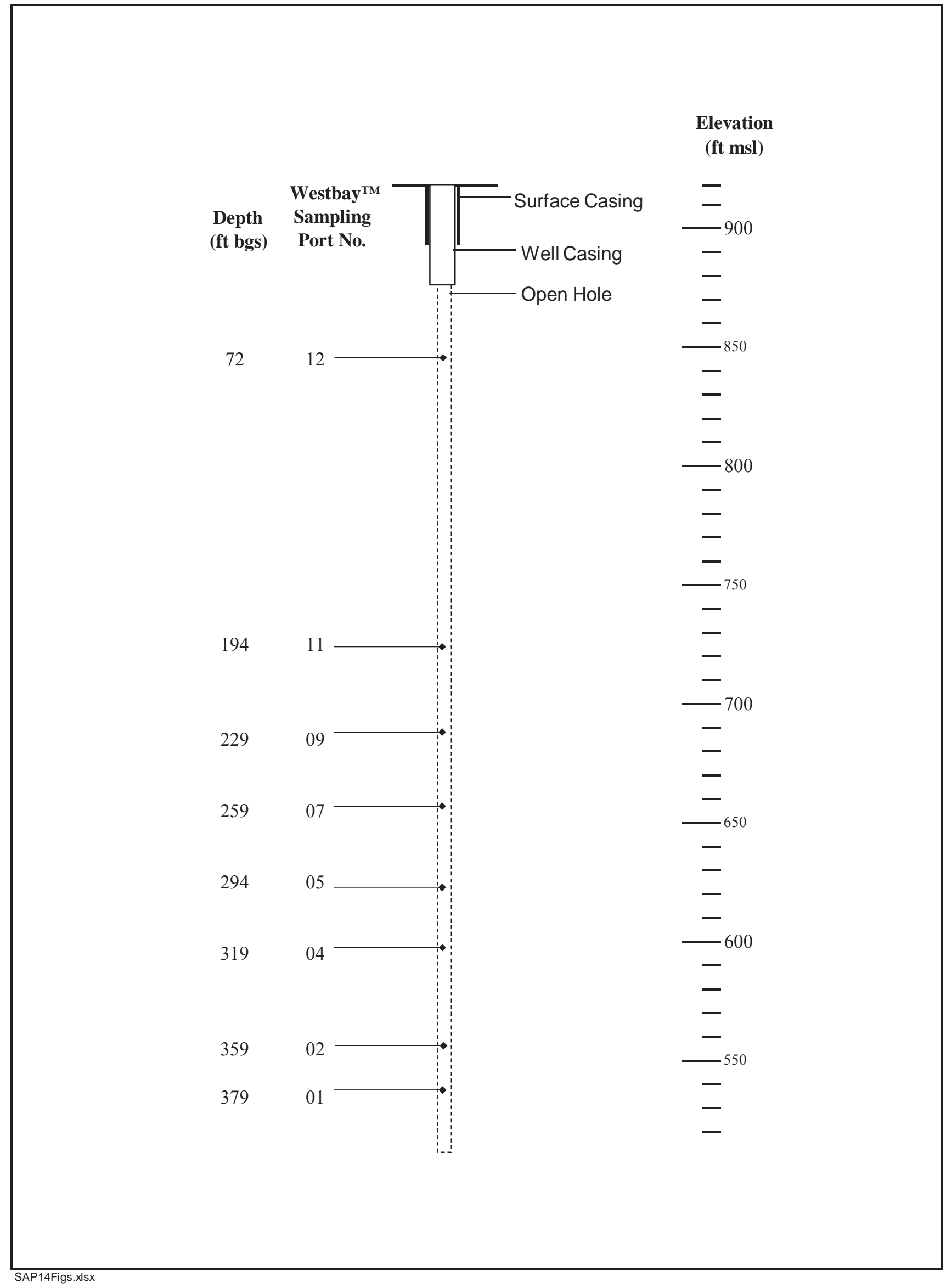

Fig. A.8. Westbay ${ }^{\mathrm{TM}}$ monitoring system sampling port depths in well GW-934. 
APPENDIX B

TABLES 
Table B.1 Sampling locations, frequency, and analytical parameters for groundwater and surface water monitoring during CY 2014

\begin{tabular}{|c|c|c|c|c|c|c|c|c|}
\hline \multirow{2}{*}{$\begin{array}{c}\text { Sampling } \\
\text { Point }^{1}\end{array}$} & \multirow{2}{*}{ Location $^{2}$} & \multirow{2}{*}{$\begin{array}{l}\text { Collection } \\
\text { Method }^{3}\end{array}$} & \multirow{2}{*}{$\begin{array}{c}\text { Sampling } \\
\text { Frequency }^{4}\end{array}$} & \multicolumn{4}{|c|}{ Samples Collected in CY $2014^{5}$} & \multirow{2}{*}{ Parameters $^{6}$} \\
\hline & & & & Q1 & Q2 & Q3 & Q4 & \\
\hline \multicolumn{9}{|c|}{ Bear Creek Hydrogeologic Regime } \\
\hline GW-006 & OLF & LFLO & Even & & & Y & & VOC(1) \\
\hline GW-014 & $\mathrm{BG}$ & LFLO & Annual & & & Y & & STD \\
\hline GW-065 & OLF & $\mathrm{NP}$ & Even & & & NP & & STD \\
\hline GW-068 & BG & LFLO & Annual & & & $\mathrm{Y}$ & & VOC(1) \\
\hline GW-082 & $\mathrm{BG}$ & LFLO & Annual & Y & & & & VOC $(1)$ \\
\hline GW-085 & OLF & LFLO & Annual & $\mathrm{D}$ & & & & Anions, RAD $(1,12)$ \\
\hline GW-098 & OLF & LFLO & Annual & & & Y & & STD \\
\hline GW-100 & S3 & LFLO & Annual & & & Y & & STD \\
\hline GW-101 & S3 & LFLO & Annual & & & Y & & STD \\
\hline GW-225 & OLF & LFLO & Annual & & & $\mathrm{D}$ & & STD \\
\hline GW-229 & OLF & LFLO & Annual & & & Y & & STD \\
\hline GW-246 & S3 & LFLO & Annual & Y & & & & STD, RAD $(3,12)$ \\
\hline GW-289 & $\mathrm{BG}$ & LFLO & Annual & Y & & & & STD \\
\hline GW-307 & $\mathrm{RS}$ & LFLO & Annual & & & Y & & VOC(1) \\
\hline GW-310 & $\mathrm{RS}$ & LFLO & Even & & & $\mathrm{D}$ & & Anions, VOC(1) \\
\hline FB-GW-312 & $\mathrm{RS}$ & & & & & $\mathrm{Y}$ & & VOC(1) \\
\hline GW-312 & $\mathrm{RS}$ & LFLO & Even & & & Y & & VOC(1) \\
\hline GW-315 & SPI & LFLO & Annual & & & $\mathrm{Y}$ & & STD \\
\hline GW-365 & OLF & LFLO & Annual & & & $\mathrm{Y}$ & & STD \\
\hline GW-526 & S3 & LFLO & Annual & & & Y & & Anions, RAD $(1,12)$ \\
\hline GW-537 & OLF & LFLO & Annual & Y & & - & & Anions, RAD $(1,12)$ \\
\hline GW-601 & OLF & LFLO & Annual & & & Y & & Anions, VOC $(1)$ \\
\hline GW-615 & S3 & LFLO & Annual & & & Y & & STD, RAD $(3,12)$ \\
\hline GW-616 & S3 & LFLO & Annual & Y & & & & STD \\
\hline GW-623 & $\mathrm{BG}$ & NP & Annual & & & NP & & $\operatorname{VOC}(1)$ \\
\hline GW-626 & $\mathrm{BG}$ & LFLO & Even & Y & & & & VOC $(1)$, MET-PMS \\
\hline GW-627 & BG & LFLO & Semiannual & Y & & Y & & VOC(1) \\
\hline GW-653 & $\mathrm{BG}$ & LFLO & Annual & & & $\mathrm{D}$ & & VOC(1) \\
\hline GW-703 & EXP-B & LFLO & Annual & & & Y & & STD \\
\hline GW-724 & EXP-C & LFLO & Annual & & & Y & & STD \\
\hline GW-725 & EXP-C & LFLO & Annual & & & $\mathrm{Y}$ & & STD \\
\hline GW-726-04 & BG & WBAY & 2014 & Y & & & & STD \\
\hline GW-726-06 & $\mathrm{BG}$ & WBAY & 2014 & $\mathrm{Y}$ & & & & STD \\
\hline GW-726-09 & $\mathrm{BG}$ & WBAY & 2014 & $\mathrm{Y}$ & & & & STD \\
\hline GW-726-12 & $\mathrm{BG}$ & WBAY & 2014 & $\mathrm{Y}$ & & & & STD \\
\hline GW-726-16 & $\mathrm{BG}$ & WBAY & 2014 & Y & & & & STD \\
\hline GW-726-20 & $\mathrm{BG}$ & WBAY & 2014 & Y & & & & STD \\
\hline GW-726-23 & BG & WBAY & 2014 & Y & & & & STD \\
\hline ER-GW-726-23 & BG & & & Y & & & & VOC(1) \\
\hline GW-738 & EXP-C & LFLO & Annual & & & $\mathrm{Y}$ & & STD \\
\hline GW-740 & EXP-C & LFLO & Annual & & & Y & & VOC(1) \\
\hline GW-829 & OLF & LFLO & Even & $\mathrm{Y}$ & & & & Anions \\
\hline
\end{tabular}


Table B.1 (continued)

\begin{tabular}{|c|c|c|c|c|c|c|c|c|}
\hline \multirow{2}{*}{$\begin{array}{c}\text { Sampling } \\
\text { Point }^{1}\end{array}$} & \multirow{2}{*}{ Location $^{2}$} & \multirow{2}{*}{$\begin{array}{l}\text { Collection } \\
\text { Method }^{3}\end{array}$} & \multirow{2}{*}{$\begin{array}{c}\text { Sampling } \\
\text { Frequency }\end{array}$} & \multicolumn{4}{|c|}{ Samples Collected in CY $2014^{5}$} & \multirow{2}{*}{ Parameters $^{6}$} \\
\hline & & & & Q1 & Q2 & Q3 & Q4 & \\
\hline \multicolumn{9}{|c|}{ Bear Creek Hydrogeologic Regime (continued) } \\
\hline BCK-04.55 & EXP-SW & GRAB & Annual & & & $\mathrm{Y}$ & & STD \\
\hline BCK-09.40 & EXP-SW & GRAB & Annual & & & Y & & STD \\
\hline BCK-11.97 & EXP-SW & GRAB & Annual & & & $\mathrm{Y}$ & & STD \\
\hline NT-01 & EXP-SW & GRAB & Annual & & & $\mathrm{Y}$ & & STD \\
\hline SS-4 & EXP-SW & GRAB & Annual & & & $\mathrm{D}$ & & STD \\
\hline SS-5 & EXP-SW & GRAB & Annual & & & $\mathrm{Y}$ & & STD \\
\hline \multicolumn{9}{|c|}{ Chestnut Ridge Hydrogeologic Regime } \\
\hline GW-322 & CRSP & LFLO & Annual & & $\mathrm{Y}$ & & & VOC(1) \\
\hline GW-608 & CRSP & LFLO & Even & & 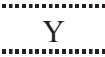 & & & VOC(1) \\
\hline GW-609 & CRSP & LFLO & Even & & Y & & & $\operatorname{VOC}(1)$ \\
\hline GW-611 & CRSP & LFLO & Even & & $\mathrm{D}$ & & & STD \\
\hline S17 & EXP-SW & $\mathrm{GRAB}$ & Annual & & Y & & & STD \\
\hline SCR1.5SW & EXP-SW & GRAB & Annual & & Y & & & STD \\
\hline SCR2.1SP & EXP-SW & GRAB & Annual & & Y & & & STD \\
\hline SCR2.2SP & EXP-SW & GRAB & Annual & & $Y$ & & & STD \\
\hline SCR3.5SW & EXP-SW & GRAB & Annual & & $\mathrm{Y}$ & & & STD \\
\hline \multicolumn{9}{|c|}{ Upper East Fork Poplar Creek Hydrogeologic Regime } \\
\hline $55-2 \mathrm{~B}$ & GRIDB3 & LFLO & Annual & & & & Y & Anions, VOC(1) \\
\hline $55-2 \mathrm{C}$ & GRIDB3 & LFLO & Annual & & & & Y... & Anions, VOC(1) \\
\hline $55-3 \mathrm{~A}$ & B9201-5 & LFLO & Annual & & & & Y... & STD \\
\hline $55-3 \mathrm{~B}$ & B9201-5 & LFLO & Annual & & & & Y & STD \\
\hline $55-3 \mathrm{C}$ & B9201-5 & LFLO & Annual & & & & Y... & STD \\
\hline $56-1 \mathrm{~A}$ & Y12 & LFLO & Annual & & & & Y & VOC(1) \\
\hline $56-1 \mathrm{C}$ & Y12 & LFLO & Annual & & & & Y & STD \\
\hline $56-2 \mathrm{~A}$ & GRIDC3 & LFLO' & Annual & & & & Y & $\mathrm{VOC}(1)$ \\
\hline $56-2 B$ & GRIDC3 & LFLO & Annual & & & & $Y$ & VOC (1) \\
\hline $56-2 C$ & GRIDC3 & LFLO & Annual & & & & Y........ & VOC(1) \\
\hline $56-3 \mathrm{~A}$ & Y12 & EFEO & Annual & & & & Y...' & VOC(1) \\
\hline $56-3 B$ & Y12 & LFLO" & Annual & & & & D....... & VOC(1) \\
\hline $56-3 C$ & $Y 12$ & LFLO & Annual & & & & Y... & VOC(1) \\
\hline $56-4 \mathrm{~A}$ & Y12 & LFLO & Annual & & & & Y... & VOC(1) \\
\hline $56-6 \mathrm{~A}$ & Y12 & LFLO & Even & & & & Y & STD \\
\hline $60-1 \mathrm{~A}$ & Y12 & LFLO & Even & & & & Y & STD \\
\hline GW-106 & S3 & LFLO & Even & & Y & & & Anions \\
\hline GW-109 & S3 & LFLO & Even & & Y & & & STD \\
\hline GW-148 & NHP & LFLO & Even & & Y & & & VOC(1) \\
\hline$G W-153$ & NHP & LFLO & Annual & Y & & & & STD \\
\hline GW-204 & T0134 & LFLO & Annual & & & Y & & STD,RAD(3) \\
\hline $\mathrm{GW}-220$ & NHP & LFLO & Semiannual & YY..." & & $\mathrm{D}$ & & VOC(1) \\
\hline GW-222 & NHP & "LFLO'" & 2014 & & & Y & & STD \\
\hline GW-240 & NHP & LFLO" & Annual & D & & & & STD \\
\hline GW-251 & S2 & LFLO" & Annual & & & Y & & Anions, VOC(1) \\
\hline GW-265 & SY & LFLO & Annual & & & & Y & VOC $(1)$ \\
\hline GW-268 & SY & LFLO & Annual & & & & Y & STD \\
\hline GW-269 & SY & LFLO & Annual & & & & Y & VOC $(1)$ \\
\hline
\end{tabular}


Table B.1 (continued)

\begin{tabular}{|c|c|c|c|c|c|c|c|c|}
\hline \multirow{2}{*}{$\begin{array}{c}\text { Sampling } \\
\text { Point }^{1}\end{array}$} & \multirow{2}{*}{ Location $^{2}$} & \multirow{2}{*}{$\begin{array}{c}\text { Collection } \\
\text { Method }^{3}\end{array}$} & \multirow{2}{*}{$\begin{array}{c}\text { Sampling } \\
\text { Frequency }\end{array}$} & \multicolumn{4}{|c|}{ Samples Collected in CY $2014^{5}$} & \multirow{2}{*}{ Parameters $^{6}$} \\
\hline & & & & Q1 & Q2 & Q3 & Q4 & \\
\hline \multicolumn{9}{|c|}{ Upper East Fork Poplar Creek Hydrogeologic Regime (continued) } \\
\hline GW-270 & SY & LFLO & Annual & & & & Y & STD \\
\hline GW-274 & SY & LFLO & Annual & & & & $\mathrm{D}$ & STD, RAD $(12)$ \\
\hline GW-275 & SY & NP & Annual & & & & NP & STD \\
\hline GW-332 & WCPA & LFLO & Annual & & & Y & & STD \\
\hline GW-337 & WCPA & LFLO & Annual & & & Y... & & Anions, VOC(1) \\
\hline GW-381 & NHP & LFLO & Annual & Y & & & & STD \\
\hline GW-383 & NHP & LFLO & Annual & Y & & & & VOC $(1)$ \\
\hline GW-619 & FTF & LFLO & Even & & $Y$ & & & $\operatorname{VOC}(1)$ \\
\hline GW-620 & FTF & LFLO & Even & & $Y$ & & & VOC(1) \\
\hline GW-633 & RG & LFLO & Annual & & & Y & & STD \\
\hline GW-656 & T0134 & LFLO & Annual & $\mathrm{D}$ & & & & $\mathrm{VOC}(1)$ \\
\hline GW-686 & CPT & LFLO & Annual & & & D & & STD \\
\hline GW-690 & CPT & LFLO & Annual & & & Y & & STD, RAD (6) \\
\hline GW-691 & CPT & LFLO & Semiannual & $Y$ & & Y... & & STD, RAD (6) \\
\hline GW-692 & CPT & LFLO & Annual & & & Y & & STD, RAD (6) \\
\hline FB-GW-698 & CPT & & & Y & & & & VOC $(1)$ \\
\hline GW-698 & B8110 & LFLO & Semiannual & Y..... & & Y & & STD, RAD 6$)$ \\
\hline GW-700 & B8110 & LFLO & Annual & Y... & & & & STD, RAD(6) \\
\hline GW-722-33 & EXP-J & WBAY & Annual & Y & & & & STD-WESTBAY \\
\hline GW-722-22 & EXP-J & WBAY & Annual & Y & & & & STD-WESTBAY \\
\hline GW-722-14 & EXP-J & WBAY & Annual & Y & & & & STD-WESTBAY \\
\hline GW-722-17 & EXP-J & WBAY & Annual & Y & & & & STD-WESTBAY \\
\hline GW-722-20 & EXP-J & WBAY" & Annual & Y & & & & STD-WESTBAY \\
\hline ER-GW-722-20 & EXP-J & & & Y & & & & VOC $(1)$ \\
\hline GW-744 & GRIDK1 & LFLO & Annual & Y... & & & & STD \\
\hline GW-747 & GRIDK2 & LFLO & Annual & Y.... & & & & STD \\
\hline GW-763 & GRIDJ3 & LFLO & Annual & Y... & & & & VOC(1) \\
\hline GW-769 & GRIDG3 & LFLO" & Semiannual & & $\mathrm{Y}$ & & Y... & STD \\
\hline GW-770 & GRIDG3 & LFLO & Annual & & $\mathrm{D}$ & & & STD \\
\hline GW-781 & GRIDE3 & LFLO" & Annual & Y & & & & STD \\
\hline GW-782 & GRIDE3 & LFLO & Annual & Y & & & & STD \\
\hline GW-783 & GRIDE3 & LFLO & Annual & Y & & & & STD \\
\hline GW-791 & GRIDD2 & LFLO & Annual & Y... & & & & STD \\
\hline GW-792 & GRIDD2 & LFLO & Even & Y & & & & VOC $(1)$ \\
\hline GW-816 & EXP-SR & LFLO & Annual & Y... & & & & STD \\
\hline GW-820 & B9201-2 & LFLO & Annual & & Y & & & STD \\
\hline GW-928 & GRIDC1 & LFLO & Even & & Y & & & STD \\
\hline GW-929 & GRIDC1 & LFLO & Even & & Y & & & STD \\
\hline GW-930 & GRIDD1 & LFLO & Even & & Y & & & STD \\
\hline GW-931 & GRIDD1 & LFLO & Even & & Y & & & STD \\
\hline
\end{tabular}


Table B.1 (continued)

\begin{tabular}{|c|c|c|c|c|c|c|c|c|}
\hline \multirow{2}{*}{$\begin{array}{c}\text { Sampling } \\
\text { Point }^{1}\end{array}$} & \multirow{2}{*}{ Location $^{2}$} & \multirow{2}{*}{$\begin{array}{l}\text { Collection } \\
\text { Method }^{3} \\
\end{array}$} & \multirow{2}{*}{$\begin{array}{c}\text { Sampling } \\
\text { Frequency }^{4}\end{array}$} & \multicolumn{4}{|c|}{ Samples Collected in CY $2014^{5}$} & \multirow{2}{*}{ Parameters $^{6}$} \\
\hline & & & & Q1 & Q2 & Q3 & Q4 & \\
\hline \multicolumn{8}{|c|}{ Upper East Fork Poplar Creek Hydrogeologic Regime (continued) } & \\
\hline GW-934-12 & NHP & WBAY & Annual & & & D & & STD-WESTBAY \\
\hline GW-934-11 & NHP & WBAY & Annual & & & Y"is & & STD-WESTBAY \\
\hline GW-934-09 & NHP & WBAY & Annual & & & 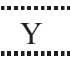 & & STD-WESTBAY \\
\hline GW-934-07 & NHP & WBAY & Annual & & & Y & & STD-WESTBAY \\
\hline GW-934-05 & NHP & WBAY & Annual & & & Y & & STD-WESTBAY \\
\hline GW-934-04 & NHP & WBAY & Annual & & & Y & & STD-WESTBAY \\
\hline GW-934-02 & NHP & WBAY & Annual & & & Y... & & STD-WESTBAY \\
\hline GW-934-01 & NHP & WBAY & Annual & & & Y & & STD-WESTBAY \\
\hline ER-GW-934-01 & NHP & & & & & Y & & $\operatorname{VOC}(1)$ \\
\hline \multicolumn{9}{|c|}{ North of Pine Ridge } \\
\hline GHK2.51WSW & EXP-SW & GRAB & Annual & & & 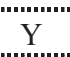 & & STD \\
\hline NPR07.0SW & EXP-SW & GRAB & Annual & & & 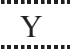 & & STD \\
\hline NPR23.0SW & EXP-SW & GRAB & Annual & & & Y & & STD \\
\hline
\end{tabular}




\section{Table B.1 (continued)}

\section{Notes:}

1 BCK - Bear Creek Kilometer (surface water station)

ER - Equipment rinsate sample

FB - Field blank sample

GW - Groundwater monitoring well

GHK - Gum Hollow Kilometer (surface water station)

NPR - North of Pine Ridge (surface water station)

NT - North Tributary to Bear Creek (surface water station)

S17 - Surface water station in SCR5

SCR - South Chestnut Ridge (spring or surface water station)

SS - Spring sampling location: South Side of Bear Creek

2 B8110 - Building 81-10

B9201-2 - Building 9201-2

B9201-5 - Building 9201-5

BG - Bear Creek Burial Grounds Waste Management Area

CPT - Coal Pile Trench

CRSP - Chestnut Ridge Security Pits

EXP - Exit Pathway Monitoring Location: Maynardville Limestone Picket B, C, or J Along Scarboro Road in the gap through Pine Ridge (-SR) Spring or Surface Water Location (-SW)

FTF - Fire Training Facility

GRID - Comprehensive Groundwater Monitoring Plan Grid Location (Martin Marietta Energy Systems, Inc. 1990)

NHP - New Hope Pond

OLF - Oil Landfarm Waste Management Area

RG - Rust Garage Area

RS - Rust Spoil Area

S2 - S-2 Site

S3 - S-3 Site

SPI - Spoil Area I

SY - Y-12 Salvage Yard

T0134 - Underground Storage Tank 0134-U

WCPA - Waste Coolant Processing Area

Y12 - Y-12 Complex

3 Sample Collection Method

LFLO - Low-flow minimal drawdown sampling

NP - No purge before sample collection; history of very low sampling rate $(<50 \mathrm{ml} / \mathrm{min})$

GRAB - Surface water sample, grab sample

WBAY - Westbay multiport method

4 Sampling Frequency: As described in the Y-12 GWPP monitoring optimization plan

(Babcock \& Wilcox Technical Services Y-12, LLC 2013)

The 2014 sampling frequency is used for wells that are sampled once every five years

5 Groundwater Monitoring Schedules (Appendix C) provide the sequence for collecting samples during each quarterly sampling event and include the waste stream identification for groundwater purged from each monitoring well. The Waste Management Plan for sampling activities is in Appendix F.

Y - Sample collection will be performed during the CY 2014 quarter

D - A field duplicate sample will collected in addition to the regular sample 


\section{Table B.1 (continued)}

\section{Notes: (continued)}

6 Table B.2 provides a comprehensive list of analytes, analytical methods, and the associated parameter group.

STD - Standard administrative parameter group, including all of the analytes in the following elementary parameter groups:

FLD - Field measurements

CHEM - Total dissolved solids, total suspended solids, and anions

MET(1) - Metals

VOC(1) - Volatile organic compounds

$\mathrm{RAD}(1)$ - Gross alpha and gross beta activity

Selective Parameter Monitoring groups (subsets of the elementary parameter groups)

Field measurements will be obtained at all locations

$\begin{aligned} & \text { Anions } \text { - Chloride, Nitrate, and Sulfate } \\ & \text { HG } \text { - Mercury } \\ & \text { MET-ICP } \text { - Metals by method SW846-6010B } \\ & \text { MET-PMS - Metals by method SW846-6020 } \\ & \text { RAD(3) - Uranium-234, -235, and -238 } \\ & \text { RAD(6) - Gamma spec for Cesium-137 } \\ & \text { RAD(12) - Technetium-99 }\end{aligned}$


Table B.2 Field measurements and laboratory analytes that comprise the elementary parameter groups for CY 2014 groundwater and surface water samples

\begin{tabular}{|c|c|c|c|c|c|}
\hline \multicolumn{2}{|c|}{$\begin{array}{c}\text { Parameter } \\
\text { Group }^{1}\end{array}$} & $\begin{array}{c}\text { Measurement or } \\
\text { Analyte }\end{array}$ & $\begin{array}{l}\text { Analytical } \\
\text { Method }^{2}\end{array}$ & $\begin{array}{l}\text { Reporting } \\
\text { Limit }^{3}\end{array}$ & Units $^{4}$ \\
\hline \multirow{6}{*}{\multicolumn{2}{|c|}{ FLD }} & Depth to Water & NA & NA & $\mathrm{ft}$ \\
\hline & & Water Temperature & NA" & NA" & centigrade \\
\hline & & $\mathrm{pH}$ & NA" & NA" & pH units \\
\hline & & Conductivity & NA & NA & $\mu \mathrm{mho} / \mathrm{cm}$ \\
\hline & & Dissolved Oxygen & "NA" & "NA" & ppm \\
\hline & & Oxidation-Reduction Potential (Redox) & NA" & NA" & $\mathrm{mV}$ \\
\hline \multirow[t]{8}{*}{ CHEM } & TDS & Total Dissolved Solids & SM 2540C 18 & 1 & $\mathrm{mg} / \mathrm{L}$ \\
\hline & TSS & Total Suspended Solids & SM 2540 D 18 & 1 & $\mathrm{mg} / \mathrm{L}$ \\
\hline & Alkalinity & Bicarbonate & SM 2320B 18 & 1 & $\mathrm{mg} / \mathrm{L}$ \\
\hline & & Carbonate & SM $2320 \mathrm{~B} 18$ & 1 & $\mathrm{mg} / \mathrm{L}$ \\
\hline & Anions & Chloride & SW846-9056 & 0.2 & $\mathrm{mg} / \mathrm{L}$ \\
\hline & & Nitrate (as Nitrogen) & EPA-353.2 1983 & 0.05 & $\mathrm{mg} / \mathrm{L}$ \\
\hline & & Sulfate & SW846-9056 & 0.25 & $\mathrm{mg} / \mathrm{L}$ \\
\hline & Fluoride & Fluoride & SM 4500F 18 & 0.1 & $\mathrm{mg} / \mathrm{L}$ \\
\hline \multirow[t]{29}{*}{ MET(1) } & MET-ICP & Aluminum & SW846-6010B & 0.2 & $\mathrm{mg} / \mathrm{L}$ \\
\hline & & Barium & SW846-6010B & 0.004 & $\mathrm{mg} / \mathrm{L}$ \\
\hline & & Beryllium & SW846-6010B & 0.0005 & $\mathrm{mg} / \mathrm{L}$ \\
\hline & & Boron & SW846-6010B & 0.1 & $\mathrm{mg} / \mathrm{L}$ \\
\hline & & Calcium & SW846-6010B & 0.2 & $\mathrm{mg} / \mathrm{L}$ \\
\hline & & Cobait & SW846-6010B & 0.02 & $\mathrm{mg} / \mathrm{L}$ \\
\hline & & Copper & SW846-6010B & 0.02 & mg/L" \\
\hline & & Iron & SW846-6010B & 0.05 & mg/L \\
\hline & & Lithium & SW846-6010B & 0.01 & mg/L" \\
\hline & & Magnesium| & SW846-6010B & 0.2 & $\mathrm{mg} / \mathrm{L}$ \\
\hline & & Manganese & SW846-6010B & 0.005 & $\mathrm{mg} / \mathrm{L}$ \\
\hline & & Molybdenum & SW846-6010B & 0.05 & mg/L \\
\hline & & Potassium & SW846-6010B & 2 & $\mathrm{mg} / \mathrm{L}$ \\
\hline & & Silver & SW846-6010B & 0.02 & mg/L" \\
\hline & & Sodium| & SW846-6010B & 0.2 & $\mathrm{mg} / \mathrm{L}$ \\
\hline & & Strontium| & SW846-6010B & 0.005 & mg/L' \\
\hline & & Thorium & SW846-6010B & 0.2 & $\mathrm{mg} / \mathrm{L}$ \\
\hline & & Vanadium & SW846-6010B & 0.02 & mg/L \\
\hline & & Zinc & SW846-6010B & 0.05 & $\mathrm{mg} / \mathrm{L}$ \\
\hline & MET-PMS & Antimony & SW846-6020 & 0.0025 & $\mathrm{mg} / \mathrm{L}$ \\
\hline & & Arsenic & SW846-6020 & 0.005 & $\mathrm{mg} / \mathrm{L}$ \\
\hline & & Cadmium & SW846-6020 & 0.0025 & $\mathrm{mg} / \mathrm{L}$ \\
\hline & & Chromium & SW846-6020 & 0.01 & $\mathrm{mg} / \mathrm{L}$ \\
\hline & & Lead & SW846-6020 & 0.0005 & mg/L \\
\hline & & Nickel| & SW846-6020 & 0.005 & $\mathrm{mg} / \mathrm{L}$ \\
\hline & & Selenium & SW846-6020 & 0.01 & $\mathrm{mg} / \mathrm{L}$ \\
\hline & & Thallium| & SW846-6020 & 0.0005 & "mg/L"' \\
\hline & & Uranium & SW846-6020 & 0.0005 & $\mathrm{mg} / \mathrm{L}$ \\
\hline & $\mathrm{HG}$ & Mercury & SW846-7470A & 0.00005 & $\mathrm{mg} / \mathrm{L}$ \\
\hline \multirow{9}{*}{\multicolumn{2}{|c|}{ VOC(1) }} & Acetone & SW846-8260B-UP & 10 & $\mu \mathrm{g} / \mathrm{L}$ \\
\hline & & Acetonitrile & SW846-8260B-UP & 5 & $\mu \mathrm{g} / \mathrm{L}$ \\
\hline & & Acrolein & SW846-8260B-UP" & 10 & $\mu \mathrm{g} / \mathrm{L}$ \\
\hline & & Acrylonitrile & SW846-8260B-UP & 5 & $\mu \mathrm{g} / \mathrm{L}$ \\
\hline & & Benzene & SW846-8260B-UP & 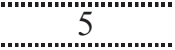 & 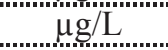 \\
\hline & & Bromochloromethane & SW846-8260B-UP & 5 & $\mu \mathrm{g} / \mathrm{L}$ \\
\hline & & Bromodichloromethane & SW846-8260B-UP & 5 & $\mu \mathrm{g} / \mathrm{L}$ \\
\hline & & Bromoform| & SW846-8260B-UP" & 5 & $\mu \mathrm{g} / \mathrm{L}$ \\
\hline & & Bromomethane & SW846-8260B-UP & 5 & $\mu \mathrm{g} / \mathrm{L}$ \\
\hline
\end{tabular}


Table B.2 (continued)

\begin{tabular}{|c|c|c|c|c|}
\hline $\begin{array}{c}\text { Parameter } \\
\text { Group }^{1}\end{array}$ & $\begin{array}{c}\text { Measurement or } \\
\text { Analyte }\end{array}$ & $\begin{array}{l}\text { Analytical } \\
\text { Method }^{2}\end{array}$ & $\begin{array}{c}\text { Reporting } \\
\text { Limit }^{3}\end{array}$ & Units $^{4}$ \\
\hline \multirow{46}{*}{$\begin{array}{l}\text { VOC(1) } \\
\text { (continued) }\end{array}$} & 2-Butanone & SW846-8260B-UP & 5 & $\mu \mathrm{g} / \mathrm{L}$ \\
\hline & Carbon disulfide & SW846-8260B-UP & 5 & $\mu \mathrm{g} / \mathrm{L}$ \\
\hline & Carbon tetrachloride & SW846-8260B-UP & 5 & $\mu \mathrm{g} / \mathrm{L}$ \\
\hline & Chlorobenzene & SW846-8260B-UP & 5 & $\mu \mathrm{g} / \mathrm{L}$ \\
\hline & Chloroethane & SW846-8260B-UP & 5 & $\mu \mathrm{g} / \mathrm{L}$ \\
\hline & 2-Chhoroethylvinyl ether & SW846-8260B-UP & 10 & $\mu \mathrm{g} / \mathrm{L}$ \\
\hline & Chloroform & SW846-8260B-UP & 5 & $\mu \mathrm{g} / \mathrm{L}$ \\
\hline & Chloromethane & SW846-8260B-UP & 5 & $\mu \mathrm{g} / \mathrm{L}$ \\
\hline & Dibromochloromethane & SW846-8260B-UP & 5 & $\mu \mathrm{g} / \mathrm{L}$ \\
\hline & 1,2-Dibromo-3-chloropropane & SW846-8260B-UP & 10 & $\mu \mathrm{gg} / \mathrm{L}$ \\
\hline & 1,2-Dibromoethane & SW846-8260B-UP & 5 & $\mu \mathrm{g} / \mathrm{L}$ \\
\hline & Dibromomethane & SW846-8260B-UP & 5 & $\mu \mathrm{g} / \mathrm{L}$ \\
\hline & 1,2-Dichlorobenzene & SW846-8260B-UP & 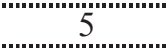 & 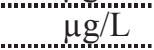 \\
\hline & 1,4-Dichlorobenzene & SW846-8260B-UP & 5 & $\mu \mathrm{g} / \mathrm{L}$ \\
\hline & 1,4-Dichloro-2-butene & SW846-8260B-UP & 5 & $\mu \mathrm{g} / \mathrm{L}$ \\
\hline & trans-1,4-Dichloro-2-butene & SW846-8260B-UP & 5 & $\mu \mathrm{g} / \mathrm{L}$ \\
\hline & Dichlorodifluoromethan & SW846-8260B-UP & 5 & $\mu \mathrm{g} / \mathrm{L}$ \\
\hline & 1,1-Dichloroethane & SW846-8260B-UP & '.5." & $\mu \mathrm{g} / \mathrm{L}$ \\
\hline & 1,2-Dichloroethane & SW846-8260B-UP & 5 & $\mu \mathrm{g} / \mathrm{L}$ \\
\hline & 1,1-Dichloroethene & SW846-8260B-UP' & - & "....." \\
\hline & cis-1,2-Dichloroethene & SW846-8260B-UP & 5 & $\mu \mathrm{g} / \mathrm{L}$ \\
\hline & trans-1,2-Dichloroethene & SW846-8260B-UP & 5 & $-\mu \mathrm{g} / \mathrm{L}$ \\
\hline & 1,2-Dichloropropane & SW846-8260B-UP & 5 & $\mu \mathrm{g} / \mathrm{L}$ \\
\hline & cis-1,3-Dichloropropene & SW846-8260B-UP & 5 & $\mu \mathrm{g} / \mathrm{L}$ \\
\hline & trans-1,3-Dichloropropene & SW846-8260B-UP & 5 & $\mu \mathrm{g} / \mathrm{L}$ \\
\hline & Ethanol & SW846-8260B-UP & 200 & $\mu \mathrm{g} / \mathrm{L}$ \\
\hline & Ethylbenzene & SW846-8260B-UP & 5 & $\mu \mathrm{g} / \mathrm{L}$ \\
\hline & Ethyl methacrylate & SW846-8260B-UP & 5 & 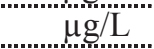 \\
\hline & 2 -Hexanone & SW846-8260B-UP & 5 & $\mu \mathrm{g} / \mathrm{L}$ \\
\hline & Iodomethane & SW846-8260B-UP & 5 & $\mu \mathrm{g} / \mathrm{L}$ \\
\hline & 4-Methyl-2-pentanone & SW846-8260B-UP & 5 & ng/L \\
\hline & Methylene chloride & SW846-8260B-UP & 3 & $\mu \mathrm{g} / \mathrm{L}$ \\
\hline & Styrene & SW846-8260B-UP & 5 & $\mu \mathrm{g} / \mathrm{L}$ \\
\hline & $1,1,1,2-T e$ etrachloroethane & SW846-8260B-UP & 5 & $\mu \mathrm{g} / \mathrm{L}$ \\
\hline & $1,1,2,2-$ Tetrachloroethane & SW846-8260B-UP & 5 & ........ \\
\hline & Tetrachloroethene & SW846-8260B-UP & 5 & $\mu \mathrm{gg} / \mathrm{L}$ \\
\hline & Toluene & SW846-8260B-UP & 5 & $\mu \mathrm{g} / \mathrm{L}$ \\
\hline & Total Xylene & SW846-8260B-UP & 5 & $\mu \mathrm{g} / \mathrm{L}$ \\
\hline & 1,1,1-Trichloroethane & SW846-8260B-UP & 5 & $\mu \mathrm{g} / \mathrm{L}$ \\
\hline & 1,1,2-Trichloroethane & SW846-8260B-UP & 5 & $\mu \mathrm{g} / \mathrm{L}$ \\
\hline & Trichloroethene & SW846-8260B-UP & 5 & $\mu \mathrm{g} / \mathrm{L}$ \\
\hline & Trichlorofluoromethane & SW846-8260B-UP & 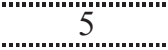 & $\mu \mathrm{g} / \mathrm{L}$ \\
\hline & 1,2,3-Trichloropropane & SW846-8260B-UP & 10 & $\mu \mathrm{g} / \mathrm{L}$ \\
\hline & 1,1,2-Trichloro-1,2,2-trifluoroethane & SW846-8260B-UP & 5 & $\mu \mathrm{g} / \mathrm{L}$ \\
\hline & Vinyl acetate & SW846-8260B-UP & 10 & $\mu \mathrm{g} / \mathrm{L}$ \\
\hline & Vinyl chloride & SW846-8260B-UP & 2 & $\mu \mathrm{g} / \mathrm{L}$ \\
\hline \multirow[t]{2}{*}{ RAD(1) } & Gross Alpha Activity & EPA-900.0 & 5 & $\mathrm{pCi} / \mathrm{L}$ \\
\hline & Gross Beta Activity & EPA-900.0 & 10 & $\mathrm{pCi} / \mathrm{L}$ \\
\hline RAD(3) & Uranium-234, $-235, \&-238$ & Y $50-A C-65-7055 \mathrm{R} 0.0$ & 0.4 & $\mathrm{pCi} / \mathrm{L}$ \\
\hline RAD(6) & Cesium-137 & Y50-AC-65-7039 & 10 & $\mathrm{pCi} / \mathrm{L}$ \\
\hline RAD(12) & Technetium-99 & Y $50-A C-65-7060$ R 0.1 & 15 & $\mathrm{pCi} / \mathrm{L}$ \\
\hline
\end{tabular}


Table B.2 (continued)

Notes:

1 Elementary Parameter Groups for the Standard Parameter Group and Selected Parameter Monitoring:

FLD - Field measurements

CHEM - Miscellaneous laboratory analytes (e.g., dissolved solids) and anions

Anions - Chloride, Nitrate, and Sulfate

MET(1) - All Metals (MET-ICP, HG, and MET-PMS)

MET-ICP - Metals by method SW846-6010B (19 metals)

$\mathrm{HG}$ - Mercury

MET-PMS - Metals by method SW846-6020 (10 metals)

VOC(1) - Volatile organic compounds (55 compounds)

RAD(1) - Gross alpha and gross beta activity

RAD(3) - Uranium-234, -235, and -238

RAD(6) - Cesium-137

RAD(12) - Technetium-99

2 Analytical Method:

NA - Not Applicable

Field measurements are performed in accordance with the following B\&W Y-12

Management Requirements operating procedures:

\begin{tabular}{|cl|cc|}
\hline Field Measurement & Procedure & Field Measurement & Procedure \\
\hline Depth to Water & Y50-71-015 & Dissolved Oxygen & Y50-71-032 \\
Water Temperature & Y50-71-030,-014 & Redox & Y50-71-033 \\
pH & Y50-71-031,-014 & Pressure Profile & Y50-71-019 \\
Conductivity & Y50-71-034,-022 & & \\
\hline
\end{tabular}

Analytical methods from:

- $\quad$ EPA - Methods for Chemical Analysis of Water and Wastes

(U.S. Environmental Protection Agency 1983)

- SM - Standard Methods for the Evaluation of Water and Wastewater, 18th Edition (American Public Health Association 1992)

- SW846 - Test Methods for Evaluating Solid Waste Physical/Chemical Methods (U.S. Environmental Protection Agency 1996)

- B\&W Y-12 ACO Procedures and laboratory test names on the table below are applicable to the analytical methods shown above in the main table:

\begin{tabular}{|cll|}
\hline Method & ACO Procedure & ACO Lab Test \\
\hline EPA-353.2 & Y50-AC-65-7659 & NO3-N \\
EPA-900.0 & Y50-AC-65-7074 & GROSSAB-ENV \\
SM 2320B 18 & Y/P65-7639 & ALKALINITY-I \\
SM 2540C 18 & Y-50-AC-65-7914 & SOLIDS-TOT-D \\
SM 2540D 18 & Y-50-AC-65-7918 & SOLIDS-TOT-S \\
SM 4500F 18 & Y-50-AC-65-7602 & FLUORIDE \\
SW846-6010B & Y50-AC-65-0040 & ICP6010 \\
SW846-6020 & Y50-AC-65-0038 & ICPMS6020EXT \\
SW846-7470A & Y50-AC-65-7470 & HGLOWRL \\
SW846-8260B-UP & Y50-AC-65-7335 & VOA8260GW \\
SW846-8260B-UP & Y50-AC-65-7335 & VOAGW-PDB \\
SW846-9056 & Y50-AC-65-7619 & ANIONS \\
Y50-AC-65-7060 & Y50-AC-65-7060 & TC99LS-ENV \\
Y50-AC-65-7039 & Y50-AC-65-7039 & GAMSPEC-ENV \\
Y50-AC-65-7061 & Y50-AC-65-7061 & ASPECU-ENV \\
\hline
\end{tabular}


Table B.2 (continued)

\section{Notes: (continued)}

3 Reporting Limits:

NA - not applicable

VOC - Reporting limits are contract-required quantitation limits; also report estimated values (with qualifier) below this limit and above the method detection limit.

RAD - Reporting limits are target minimum detectable activities (MDAs) that may be obtained under optimal analytical conditions; actual MDAs are sample-specific and may vary significantly from the target value.

4 Units:
$\mathrm{mg} / \mathrm{L} \quad$ - milligrams per liter
$\mathrm{mV}$ - millivolts
NTU - nephelometric turbidity units
ppm - parts per million
pCi/L - picoCuries per liter 
APPENDIX C

CY 2014 GROUNDWATER MONITORING SCHEDULES (Insert When Issued, Before Each Quarterly Sampling Event) 
APPENDIX D

ADDENDA TO THE CY 2014 SAMPLING AND ANALYSIS PLAN (if issued) 
APPENDIX E

LABORATORY REQUIREMENTS

(Bottle Lists, Holding Times, Turnaround Time, Elevated Minimum Activity) 


\section{CY 2014 SAP BOTTLE LISTS}

\section{STD}

\begin{tabular}{||l|c|c|l||}
\hline \multicolumn{1}{|c|}{ Parameter } & Lab Tests & $\begin{array}{c}\text { Chemical } \\
\text { Preservative }\end{array}$ & \multicolumn{1}{|c||}{ Bottle Types/Size } \\
\hline \hline $\begin{array}{l}\text { Anions, Fluoride, } \\
\text { Carbonate and } \\
\text { Bicarbonate }\end{array}$ & $\begin{array}{c}\text { ANIONS, } \\
\text { FLUORIDE, } \\
\text { ALKALINITY-I }\end{array}$ & None & $1-250 \mathrm{~mL}$ polyethylene \\
\hline Nitrate & NO3-N & $\begin{array}{c}\mathrm{H}_{2} \mathrm{SO}_{4} \text { to } \mathrm{pH}< \\
2 ; 4^{\mathrm{O}}+/-2^{\mathrm{O}}\end{array}$ & $1-100 \mathrm{~mL}$ polyethylene \\
\hline Total Suspended Solids & SOLIDS-TOT-S & None & $1-250 \mathrm{~mL}$ polyethylene \\
\hline Total Dissolved Solids & SOLIDS-TOT-D & None & $1-250 \mathrm{~mL}$ polyethylene \\
\hline $\begin{array}{l}\text { Total Metals (ICP,ICP- } \\
\text { MS, and Hg) }\end{array}$ & $\begin{array}{c}\text { ICP6010, } \\
\text { ICPMS6020-EXT, } \\
\text { HGLOWRL }\end{array}$ & $\mathrm{HNO}_{3}$ & $1-500 \mathrm{~mL}$ polyethylene \\
\hline Radiochemistry (UV) & GROSSAB-ENV & $\mathrm{HNO}_{3}$ & $1-1 \mathrm{~L}$ polyethylene \\
\hline Volatiles & VOA8260GW & None & $\begin{array}{l}2-40 \mathrm{~mL} \text { amber glass } \\
\text { with Teflon lined septum } \\
\text { lids }\end{array}$ \\
\hline $\begin{array}{l}\text { Trip Blank (VOA) } \\
\text { (one per cooler) }\end{array}$ & VOA8260GW & None & $\begin{array}{l}1-40 \mathrm{~mL} \text { amber glass } \\
\text { with Teflon lined septum } \\
\text { lid }\end{array}$ \\
\hline
\end{tabular}

\section{STD: $\quad$ LIMS LAB TEST ID}

CHEM ALKALINITY-I, ANIONS, NO3-N, FLUORIDE, SOLIDS-TOT-S, SOLIDS-TOT-D MET(1) ICP6010, ICPMS6020-EXT and HGLOWRL

VOC(1) VOA8260GW

RAD(1)GROSSAB-ENV

${ }^{1}$ All samples chilled to $4{ }^{\circ} \mathrm{C}+/-2^{\circ} \mathrm{C}$ 


\section{CY 2014 SAP BOTTLE LISTS}

\section{STD-WESTBAY}

\begin{tabular}{||l|c|c|l||}
\hline \multicolumn{1}{|c|}{ Parameter } & Lab Tests & $\begin{array}{c}\text { Chemical } \\
\text { Preservative }\end{array}$ & \multicolumn{1}{|c|}{ Bottle Types/Size } \\
\hline $\begin{array}{l}\text { Anions, Fluoride, } \\
\text { Carbonate and } \\
\text { Bicarbonate }\end{array}$ & $\begin{array}{c}\text { ANIONS, } \\
\text { FLUORIDE, } \\
\text { ALKALINITY-I }\end{array}$ & None & $1-250 \mathrm{~mL}$ polyethylene \\
\hline Nitrate & NO3-N & $\begin{array}{c}\mathrm{H}_{2} \mathrm{SO}_{4} \text { to } \mathrm{pH}< \\
2 ; 4^{\circ}+/ 2^{\mathrm{O}}\end{array}$ & $1-100 \mathrm{~mL}$ polyethylene \\
\hline Total Suspended Solids & SOLIDS-TOT-S & None & $1-250 \mathrm{~mL}$ polyethylene \\
\hline Total Dissolved Solids & SOLIDS-TOT-D & None & $1-250 \mathrm{~mL}$ polyethylene \\
\hline $\begin{array}{l}\text { Total Metals (ICP,ICP- } \\
\text { MS, and Hg) }\end{array}$ & $\begin{array}{c}\text { ICP6010, } \\
\text { ICPS6020-EXT, } \\
\text { HGLOWRL }\end{array}$ & $\mathrm{HNO}_{3}$ & $1-250 \mathrm{~mL}$ polyethylene \\
\hline Radiochemistry (UV) & $\begin{array}{c}\text { GROSSAB-ENV } \\
\mathrm{HNO}_{3}\end{array}$ & $\begin{array}{l}1-500 \mathrm{~mL} \\
\text { polyethylene }\end{array}$ \\
\hline Volatiles & VOA8260GW & None & $\begin{array}{l}2-40 \mathrm{~mL} \text { amber glass } \\
\text { with Teflon lined septum } \\
\text { lids }\end{array}$ \\
\hline $\begin{array}{l}\text { Trip Blank (VOA) } \\
\text { (one per cooler) }\end{array}$ & VOA8260GW & None & $\begin{array}{l}1-40 \mathrm{~mL} \text { amber glass } \\
\text { with Teflon lined septum } \\
\text { lid }\end{array}$ \\
\hline
\end{tabular}

\section{STD: $\quad$ LIMS LAB TEST ID}

CHEM ALKALINITY-I, ANIONS, NO3-N, FLUORIDE, SOLIDS-TOT-S, SOLIDS-TOT-D MET(1) ICP6010, ICPMS6020-EXT and HGLOWRL

VOC(1) VOA8260GW

RAD(1)GROSSAB-ENV

${ }^{1}$ All samples chilled to $4{ }^{\circ} \mathrm{C}+/-2^{\circ} \mathrm{C}$ 


\section{CY 2014 SAP BOTTLE LISTS}

\section{STD, RAD (3)}

\begin{tabular}{|c|c|c|c|}
\hline Parameter & Lab Tests & $\begin{array}{c}\text { Chemical } \\
\text { Preservative }^{1}\end{array}$ & Bottle Types/Size \\
\hline $\begin{array}{l}\text { Anions, Fluoride, } \\
\text { Carbonate and } \\
\text { Bicarbonate }\end{array}$ & $\begin{array}{l}\text { ANIONS, } \\
\text { FLUORIDE, } \\
\text { ALKALINITY-I }\end{array}$ & None & 1 - $250 \mathrm{~mL}$ polyethylene \\
\hline Nitrate & NO3-N & $\begin{array}{c}\mathrm{H}_{2} \mathrm{SO}_{4} \text { to } \mathrm{pH}< \\
2 ; 4^{\mathrm{O}}+1-2^{\mathrm{O}}\end{array}$ & 1-100 mL polyethylene \\
\hline Total Suspended Solids & SOLIDS-TOT-S & None & 1 - $250 \mathrm{~mL}$ polyethylene \\
\hline Total Dissolved Solids & SOLIDS-TOT-D & None & 1 - $250 \mathrm{~mL}$ polyethylene \\
\hline $\begin{array}{l}\text { Total Metals (ICP,ICP- } \\
\text { MS, and Hg) }\end{array}$ & $\begin{array}{l}\text { ICP6010, } \\
\text { ICPMS6020-EXT, } \\
\text { HGLOWRL }\end{array}$ & $\mathrm{HNO}_{3}$ & 1 - $500 \mathrm{~mL}$ polyethylene \\
\hline Radiochemistry (UV) & $\begin{array}{l}\text { GROSSAB-ENV } \\
\text { ASPECU-ENV }\end{array}$ & $\mathrm{HNO}_{3}$ & $1-1 \mathrm{~L}$ polyethylene \\
\hline Volatiles & VOA8260GW & None & $\begin{array}{l}2-40 \mathrm{~mL} \text { amber glass } \\
\text { with Teflon lined septum } \\
\text { lids }\end{array}$ \\
\hline $\begin{array}{l}\text { Trip Blank (VOA) } \\
\text { (one per cooler) }\end{array}$ & VOA8260GW & None & $\begin{array}{l}1-40 \mathrm{~mL} \text { amber glass } \\
\text { with Teflon lined septum } \\
\text { lid }\end{array}$ \\
\hline
\end{tabular}

STD: $\quad$ LIMS LAB TEST ID

CHEM ALKALINITY-I, ANIONS, NO3-N, FLUORIDE, SOLIDS-TOT-S, SOLIDS-TOT-D

MET(1) ICP6010, ICPMS6020-EXT and HGLOWRL

VOC(1) VOA8260GW

RAD(1)GROSSAB-ENV

RAD(3)ASPECU-ENV

${ }^{1}$ All samples chilled to $4^{\circ} \mathrm{C}+/-2^{\circ} \mathrm{C}$ 


\section{CY 2014 SAP BOTTLE LISTS \\ STD, RAD (12)}

\begin{tabular}{||l|c|c|l||}
\hline \multicolumn{1}{|c|}{ Parameter } & Lab Tests & $\begin{array}{c}\text { Chemical } \\
\text { Preservative }\end{array}$ & \multicolumn{1}{|c||}{ Bottle Types/Size } \\
\hline \hline $\begin{array}{l}\text { Anions, Fluoride, } \\
\text { Carbonate and } \\
\text { Bicarbonate }\end{array}$ & $\begin{array}{c}\text { ANIONS, } \\
\text { FLUORIDE, } \\
\text { ALKALINITY-I }\end{array}$ & None & $1-250 \mathrm{~mL}$ polyethylene \\
\hline Nitrate & NO3-N & $\begin{array}{c}\mathrm{H}_{2} \mathrm{SO}_{4} \text { to } \mathrm{pH}< \\
2 ; 4^{\mathrm{O}}+/ 2^{\mathrm{O}}\end{array}$ & $1-100 \mathrm{~mL}$ polyethylene \\
\hline Total Suspended Solids & SOLIDS-TOT-S & None & $1-250 \mathrm{~mL}$ polyethylene \\
\hline Total Dissolved Solids & $\begin{array}{c}\text { SOLIDS-TOT-D } \\
\text { MS, and Hg) }\end{array}$ & None & $1-250 \mathrm{~mL}$ polyethylene \\
\hline $\begin{array}{l}\text { ICP6010, } \\
\text { ICPMS6020-EXT, } \\
\text { HGLOWRL }\end{array}$ & $\mathrm{HNO}_{3}$ & $1-500 \mathrm{~mL}$ polyethylene \\
\hline $\begin{array}{l}\text { GROSSAB-ENV } \\
\text { TC99LS-ENV }\end{array}$ & $\mathrm{HNO}_{3}$ & $1-1 \mathrm{~L}$ polyethylene \\
\hline $\begin{array}{l}\text { Volatiles } \\
\text { Trip Blank (VOA) } \\
\text { (one per cooler) }\end{array}$ & $\begin{array}{l}\text { VOA8260GW } \\
\text { VOA8260GW }\end{array}$ & None & $\begin{array}{l}2-40 \mathrm{~mL} \text { amber glass } \\
\text { with Teflon lined septum } \\
\text { lids }\end{array}$ \\
\hline \hline
\end{tabular}

STD: $\quad$ LIMS LAB TEST ID

CHEM ALKALINITY-I, ANIONS, NO3-N, FLUORIDE, SOLIDS-TOT-S, SOLIDS-TOT-D

MET(1) ICP6010, ICPMS6020-EXT and HGLOWRL

VOC(1) VOA8260GW

RAD(1)GROSSAB-ENV

RAD(12) TC99LS-ENV

${ }^{1}$ All samples chilled to $4^{\circ} \mathrm{C}+/-2^{\circ} \mathrm{C}$ 


\section{CY 2014 SAP BOTTLE LISTS \\ STD, RAD $(3,12)$}

\begin{tabular}{|c|c|c|c|}
\hline Parameter & Lab Tests & $\begin{array}{c}\text { Chemical }^{1} \\
\text { Preservative }^{1}\end{array}$ & Bottle Types/Size \\
\hline $\begin{array}{l}\text { Anions, Fluoride, } \\
\text { Carbonate and } \\
\text { Bicarbonate }\end{array}$ & $\begin{array}{l}\text { ANIONS, } \\
\text { FLUORIDE, } \\
\text { ALKALINITY-I }\end{array}$ & None & 1 - $250 \mathrm{~mL}$ polyethylene \\
\hline Nitrate & NO3-N & $\begin{array}{c}\mathrm{H}_{2} \mathrm{SO}_{4} \text { to } \mathrm{pH}< \\
2 ; 4^{\mathrm{O}}+1-2^{\mathrm{O}}\end{array}$ & 1 - $100 \mathrm{~mL}$ polyethylene \\
\hline Total Suspended Solids & SOLIDS-TOT-S & None & 1 - $250 \mathrm{~mL}$ polyethylene \\
\hline Total Dissolved Solids & SOLIDS-TOT-D & None & 1 - $250 \mathrm{~mL}$ polyethylene \\
\hline $\begin{array}{l}\text { Total Metals (ICP,ICP- } \\
\text { MS, and Hg) }\end{array}$ & $\begin{array}{l}\text { ICP6010, } \\
\text { ICPMS6020-EXT, } \\
\text { HGLOWRL }\end{array}$ & $\mathrm{HNO}_{3}$ & 1 - $500 \mathrm{~mL}$ polyethylene \\
\hline Radiochemistry (UV) & $\begin{array}{c}\text { GROSSAB-ENV } \\
\text { TC99LS-ENV } \\
\text { ASPECU-ENV }\end{array}$ & $\mathrm{HNO}_{3}$ & 1 - 1 L polyethylene \\
\hline Volatiles & VOA8260GW & None & $\begin{array}{l}2-40 \mathrm{~mL} \text { amber glass } \\
\text { with Teflon lined septum } \\
\text { lids }\end{array}$ \\
\hline $\begin{array}{l}\text { Trip Blank (VOA) } \\
\text { (one per cooler) }\end{array}$ & VOA8260GW & None & $\begin{array}{l}1 \text { - } 40 \mathrm{~mL} \text { amber glass } \\
\text { with Teflon lined septum } \\
\text { lid }\end{array}$ \\
\hline
\end{tabular}

STD: $\quad$ LIMS LAB TEST ID

CHEM ALKALINITY-I, ANIONS, NO3-N, FLUORIDE, SOLIDS-TOT-S, SOLIDS-TOT-D

MET(1) ICP6010, ICPMS6020-EXT and HGLOWRL

VOC(1) VOA8260GW

RAD(1) GROSSAB-ENV

RAD(3)ASPECU-ENV

RAD(12) TC99LS-ENV 


\section{CY 2014 SAP BOTTLE LISTS}

\section{STD, RAD (6)}

\begin{tabular}{|c|c|c|c|}
\hline Parameter & Lab Tests & $\begin{array}{c}\text { Chemical }^{\text {Cheservative }}{ }^{1} \\
\text { Preser }^{2}\end{array}$ & Bottle Types/Size \\
\hline $\begin{array}{l}\text { Anions, Fluoride, } \\
\text { Carbonate and } \\
\text { Bicarbonate }\end{array}$ & $\begin{array}{l}\text { ANIONS, } \\
\text { FLUORIDE, } \\
\text { ALKALINITY-I }\end{array}$ & None & 1 - 250 mL polyethylene \\
\hline Nitrate & NO3-N & $\begin{array}{c}\mathrm{H}_{2} \mathrm{SO}_{4} \text { to } \mathrm{pH}< \\
2 ; 4^{\mathrm{O}}+1-2^{\mathrm{O}}\end{array}$ & 1 - $100 \mathrm{~mL}$ polyethylene \\
\hline Total Suspended Solids & SOLIDS-TOT-S & None & 1 - 250 mL polyethylene \\
\hline Total Dissolved Solids & SOLIDS-TOT-D & None & 1 - 250 mL polyethylene \\
\hline $\begin{array}{l}\text { Total Metals (ICP,ICP- } \\
\text { MS, and Hg) }\end{array}$ & $\begin{array}{l}\text { ICP6010, } \\
\text { ICPMS6020-EXT, } \\
\text { HGLOWRL }\end{array}$ & $\mathrm{HNO}_{3}$ & 1 - $500 \mathrm{~mL}$ polyethylene \\
\hline Radiochemistry (UV) & GAMSPEC-ENV & $\mathrm{HNO}_{3}$ & 1-1 L polyethylene \\
\hline Volatiles & VOA8260GW & None & $\begin{array}{l}2 \text { - } 40 \mathrm{~mL} \text { amber glass } \\
\text { with Teflon lined septum } \\
\text { lids }\end{array}$ \\
\hline $\begin{array}{l}\text { Trip Blank (VOA) } \\
\text { (one per cooler) }\end{array}$ & VOA8260GW & None & $\begin{array}{l}1-40 \mathrm{~mL} \text { amber glass } \\
\text { with Teflon lined septum } \\
\text { lid }\end{array}$ \\
\hline
\end{tabular}

STD:

LIMS LAB TEST ID

CHEM ALKALINITY-I, ANIONS, NO3-N, FLUORIDE, SOLIDS-TOT-S, SOLIDS-TOT-D

MET(1) ICP6010, ICPMS6020-EXT and HGLOWRL

VOC(1) VOA8260GW

RAD(6) GAMSPEC-ENV

${ }^{1}$ All samples chilled to $4^{\circ} \mathrm{C}+/-2^{\circ} \mathrm{C}$ 


\section{CY 2014 SAP BOTTLE LISTS}

\section{Anions}

\begin{tabular}{||l|c|c|c||}
\hline \multicolumn{1}{|c|}{ Parameter } & Lab Tests & $\begin{array}{c}\text { Chemical } \\
\text { Preservative }\end{array}$ & Bottle Types/Size \\
\hline \hline Anions & ANIONS & None & $1-250 \mathrm{~mL}$ polyethylene \\
\hline \hline Nitrate & $\mathrm{NO}-\mathrm{N}$ & $\begin{array}{c}\mathrm{H}_{2} \mathrm{SO}_{4} \text { to } \mathrm{pH}< \\
2 ; 4^{\mathrm{O}}+/-2^{\mathrm{O}}\end{array}$ & $1-100 \mathrm{~mL}$ polyethylene \\
\hline
\end{tabular}

Parameter: $\quad$ LIMS LAB TEST ID

Anions ANIONS

Nitrate NO3-N

${ }^{1}$ All samples chilled to $4{ }^{\circ} \mathrm{C}+/-2^{\circ} \mathrm{C}$ 


\section{CY 2014 SAP BOTTLE LISTS}

\section{Anions, VOC (1)}

\begin{tabular}{||l|c|c|c||}
\hline \multicolumn{1}{|c|}{ Parameter } & Lab Tests & $\begin{array}{c}\text { Chemical } \\
\text { Preservative }\end{array}$ & Bottle Types/Size \\
\hline \hline Anions & ANIONS & None & $1-250 \mathrm{~mL}$ polyethylene \\
\hline \hline Nitrate & NO3-N & $\begin{array}{c}\mathrm{H}_{2} \mathrm{SO}_{4} \text { to } \mathrm{pH}< \\
2 ; 4^{\mathrm{O}}+/ 2^{\mathrm{O}}\end{array}$ & $1-100 \mathrm{~mL}$ polyethylene \\
\hline \hline Volatiles & VOA8260GW & None & $\begin{array}{l}2-40 \mathrm{~mL} \text { amber glass } \\
\text { with Teflon lined septum } \\
\text { lids }\end{array}$ \\
\hline
\end{tabular}

\section{Parameter:}

Anions

Nitrate

$\operatorname{VOC}(1)$

\section{LIMS LAB TEST ID}

ANIONS

NO3-N

VOA8260GW

${ }^{1}$ All samples chilled to $4^{\circ} \mathrm{C}+/-2^{\circ} \mathrm{C}$ 


\section{CY 2014 SAP BOTTLE LISTS}

Anions, RAD $(1,12)$

\begin{tabular}{||l|c|c|c||}
\hline \multicolumn{1}{|c|}{ Parameter } & Lab Tests & $\begin{array}{c}\text { Chemical } \\
\text { Preservative }\end{array}$ & \multicolumn{1}{||}{ Bottle Types/Size } \\
\hline \hline Anions & ANIONS & None & $1-250 \mathrm{~mL}$ polyethylene \\
\hline Nitrate & $\mathrm{NO3-N}$ & $\begin{array}{c}\mathrm{H}_{2} \mathrm{SO}_{4} \text { to } \mathrm{pH}< \\
2 ; 4^{\mathrm{O}}+/-2^{\mathrm{O}}\end{array}$ & $1-100 \mathrm{~mL}$ polyethylene \\
\hline $\begin{array}{l}\text { Gross Alpha/Beta and } \\
\text { Tc-99 }\end{array}$ & $\begin{array}{c}\text { GROSSAB-ENV } \\
\text { TC99LS-ENV }\end{array}$ & $\mathrm{HNO}_{3}$ & $1-1 \mathrm{~L}$ polyethylene \\
\hline
\end{tabular}

\section{Parameters:}

Anions

Nitrate

$\mathrm{RAD}(1)$

$\operatorname{RAD}(12)$

\section{LIMS LAB TEST ID}

ANIONS

NO3-N

GROSSAB-ENV

TC99LS-ENV

${ }^{1} \mathrm{All}$ samples chilled to $4^{\circ} \mathrm{C}+/-2^{\circ} \mathrm{C}$ 


\section{CY 2014 SAP BOTTLE LISTS}

$\operatorname{VOC}(1)$

\begin{tabular}{||c|c|c|c||}
\hline \multicolumn{1}{|c|}{ Parameter } & Lab Tests & $\begin{array}{c}\text { Chemical } \\
\text { Preservative }\end{array}$ & Bottle Types/Size \\
\hline \hline Volatiles & VOA8260GW & None & $\begin{array}{l}2-40 \mathrm{~mL} \text { amber glass } \\
\text { with Teflon lined septum } \\
\text { lids }\end{array}$ \\
\hline
\end{tabular}

Parameter:

LIMS LAB TEST ID

$\operatorname{VOC}(1)$

VOA8260GW

${ }^{1}$ All samples chilled to $4^{\circ} \mathrm{C}+/-2^{\circ} \mathrm{C}$ 


\section{CY 2014 SAP BOTTLE LISTS}

\section{VOC (1), MET-PMS}

\begin{tabular}{||l|c|c|c||}
\hline \multicolumn{1}{|c|}{ Parameter } & Lab Tests & $\begin{array}{c}\text { Chemical } \\
\text { Preservative }\end{array}$ & \multicolumn{1}{||}{ Bottle Types/Size } \\
\hline \hline MET-PMS (ICP-MS) & ICPMS6020-EXT & $\mathrm{HNO}_{3}$ & $1-250 \mathrm{~mL}$ polyethylene \\
\hline Volatiles & VOA8260GW & None & $\begin{array}{l}2-40 \mathrm{~mL} \text { amber glass } \\
\text { with Teflon lined septum } \\
\text { lids }\end{array}$ \\
\hline
\end{tabular}

Parameters: LIMS LAB TEST ID

MET-PMS ICPMS6020-EXT

VOC(1) VOA8260GW

${ }^{1}$ All samples chilled to $4{ }^{\circ} \mathrm{C}+/-2^{\circ} \mathrm{C}$ 


\section{ESTABLISHED HOLDING TIMES}

\begin{tabular}{|l|l|}
\hline Parameter & Holding Times \\
\hline Alkalinity (Carbonate, Bicarbonate) & 14 days \\
\hline Anions (Chloride, Nitrate, and Sulfate) & 28 days \\
\hline Fluoride & 28 days \\
\hline Mercury & 28 days \\
\hline Metals (ICP, ICPMS) & 6 months \\
\hline Radiochemistry & 6 months \\
\hline Solids, Total Dissolved & 7 days \\
\hline Solids, Total Suspended & 7 days \\
\hline VOA & 7 days \\
\hline
\end{tabular}

\section{ESTABLISHED TURNAROUND TIMES}

The Groundwater Protection Program and the Analytical Chemistry Organization (ACO) laboratory have agreed upon a turnaround time, such that the analytical data generated from each sampling location will be completed within 35 days of receipt. Every two weeks, data that has been approved since the previous twoweek period will be transmitted in the form of hard copy of the approved lab reports for each location, along with an electronic copy in a standardized and compatible format (please see the most recent version of the Y-12 Plant Groundwater Protection Program Data Management Plan).

\section{ELEVATED MINIMUM DETECTABLE ACTIVITY}

Groundwater samples with high TDS (>1,000 mg/L) typically have elevated minimum detectable activities (MDAs) for gross alpha ( $>15 \mathrm{pCi} / \mathrm{L})$ and gross beta (> $50 \mathrm{pCi} / \mathrm{L})$. However, the MDAs for specific isotopic analyses are unaffected by the sample solid content. For samples with gross activity results that are less than an elevated MDA, and specific isotopic analyses have not been requested, the laboratory will issue a request to analyze for the principal alpha- or beta-emitting isotopes. That is, if the gross alpha MDA exceeds $15 \mathrm{pCi} / \mathrm{L}$ and the result is less than $15 \mathrm{pCi} / \mathrm{L}$, then the laboratory will request analyses of isotopic uranium (by method Y/P65-7061). Similarly, if a sample has an elevated gross beta MDA ( $>50 \mathrm{pCi} / \mathrm{L})$ and the result is less than the MDA, then the laboratory would request analysis of technetium-99 activity. These requests will be approved by the Y-12 Groundwater Protection Program manager, or designee, before analyses are performed. 
APPENDIX F

MANAGEMENT OF PURGED GROUNDWATER 
APPENDIX F.1

WASTE MANAGEMENT PLAN 
Y/TS-2319

\title{
WASTE MANAGEMENT PLAN for Waste Streams generated from Y-12 Groundwater Protection Program Sampling Activities
}

Date Issued - 08/27/2012

\author{
prepared by: \\ Y-12 Groundwater Protection Program \\ Environmental Compliance Department \\ Y-12 National Security Complex \\ P.O. Box 2009 \\ Oak Ridge, TN 37831 \\ managed by: \\ B\&W Y-12, LLC \\ for the: \\ U.S. Department of Energy \\ Under Contract Number: \\ DE-AC05-00OR22800
}

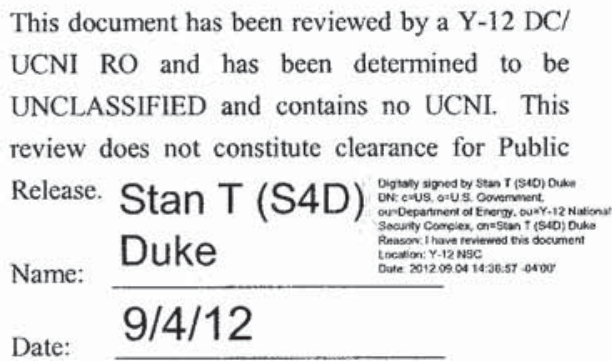

F1.1-1 


\section{Approvals}

Cheryl LaBorde

$B \& W$ Y-12, LLC

Waste Engineer

Brad Skaggs or Mark Burris

$B \& W Y-12, L L C$

Environmental Compliance

Steve Field

$B \& W Y-12, L L C$

Environmental Officer

Jan Jackson or Jeannette Widman $B \& W Y-12, L L C$

Sustainability and Stewardship

Prepared by Stacy Jollay

$B \& W Y-12, L L C$

Environmental Compliance labordecm@ Digtaly signed by

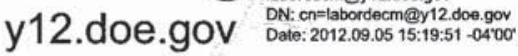
Date

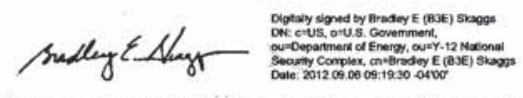

Date

fieldsm@y12 Dightally signed by

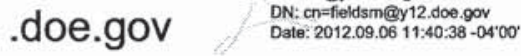

Date

widmanjl@y1 1 Digitally signed by

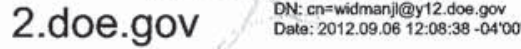

Date
Jollay

Date 
Introduction

The Y-12 National Security Complex (Y-12) Groundwater Protection Program (GWPP) performs surveillance monitoring across Y-12. On average 110 locations are sampled annually. Due to legacy operations and waste management practices numerous groundwater contaminants are present across $\mathrm{Y}-12$. In order to sample a groundwater well purged water from surveillance monitoring activities becomes waste.

The U. S. Department of Energy (DOE) order groundwater surveillance sampling performed by the Y12 GWPP has been determined to fall under the Resource Conservation and Recovery Act (RCRA) regulations for waste disposal.

As part of the annual preparation of the Sampling Analysis Plan (SAP) historical sampling data from groundwater locations is reviewed. This review verifies the concentrations such that constituent increases or spikes are considered in waste characterization. This ensures that current and correct waste codes are used. In addition to the annual review, quarterly reviews are done for further verification as part of the development of the quarterly groundwater sampling schedule. 


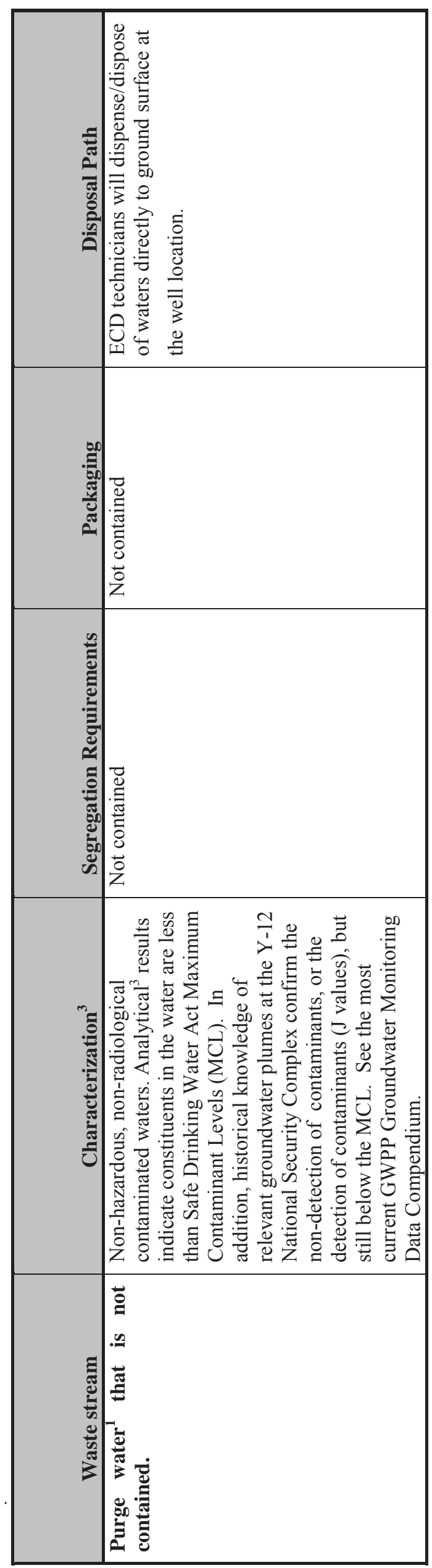

F1.1-4 


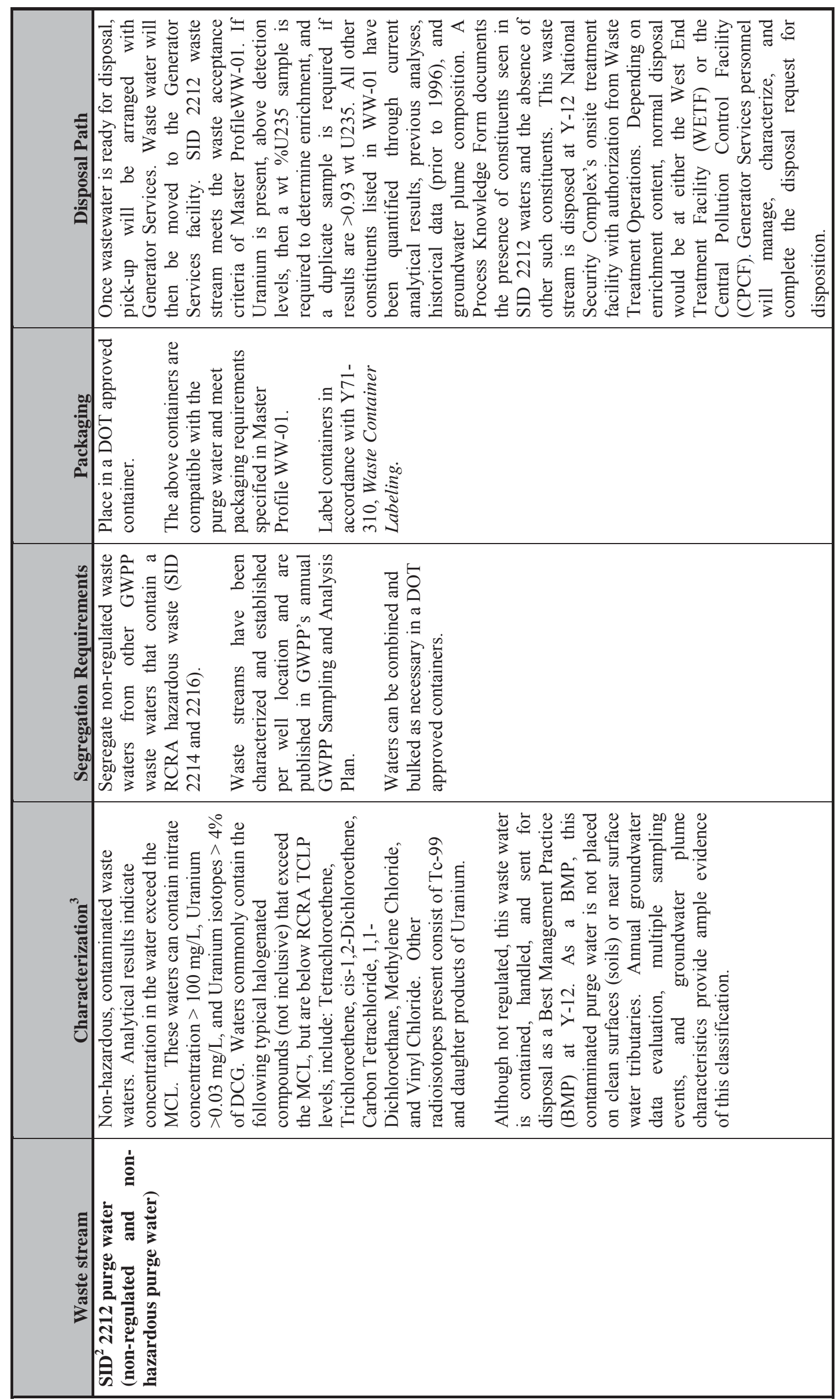

F1.1-5 


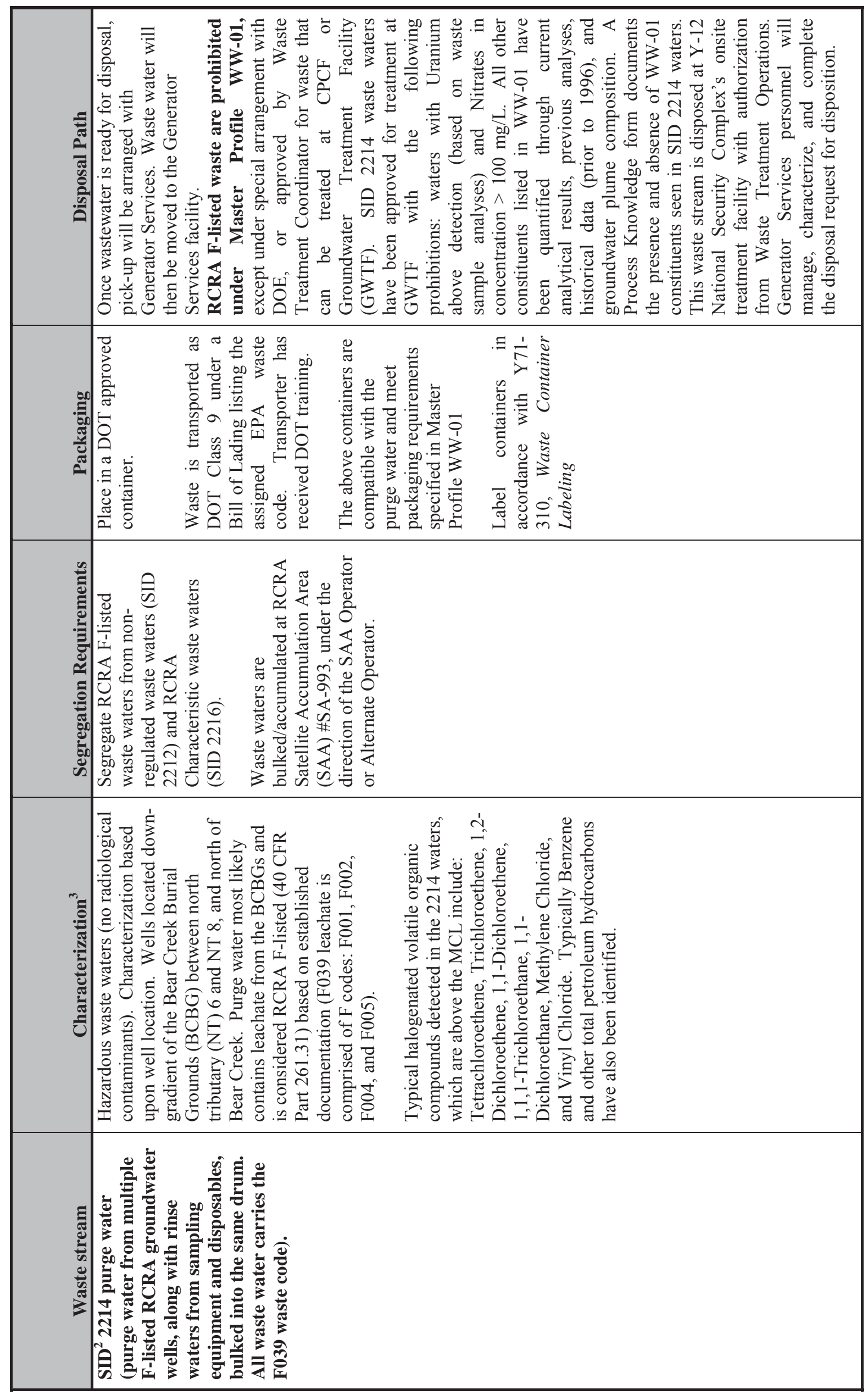

F1.1-6 


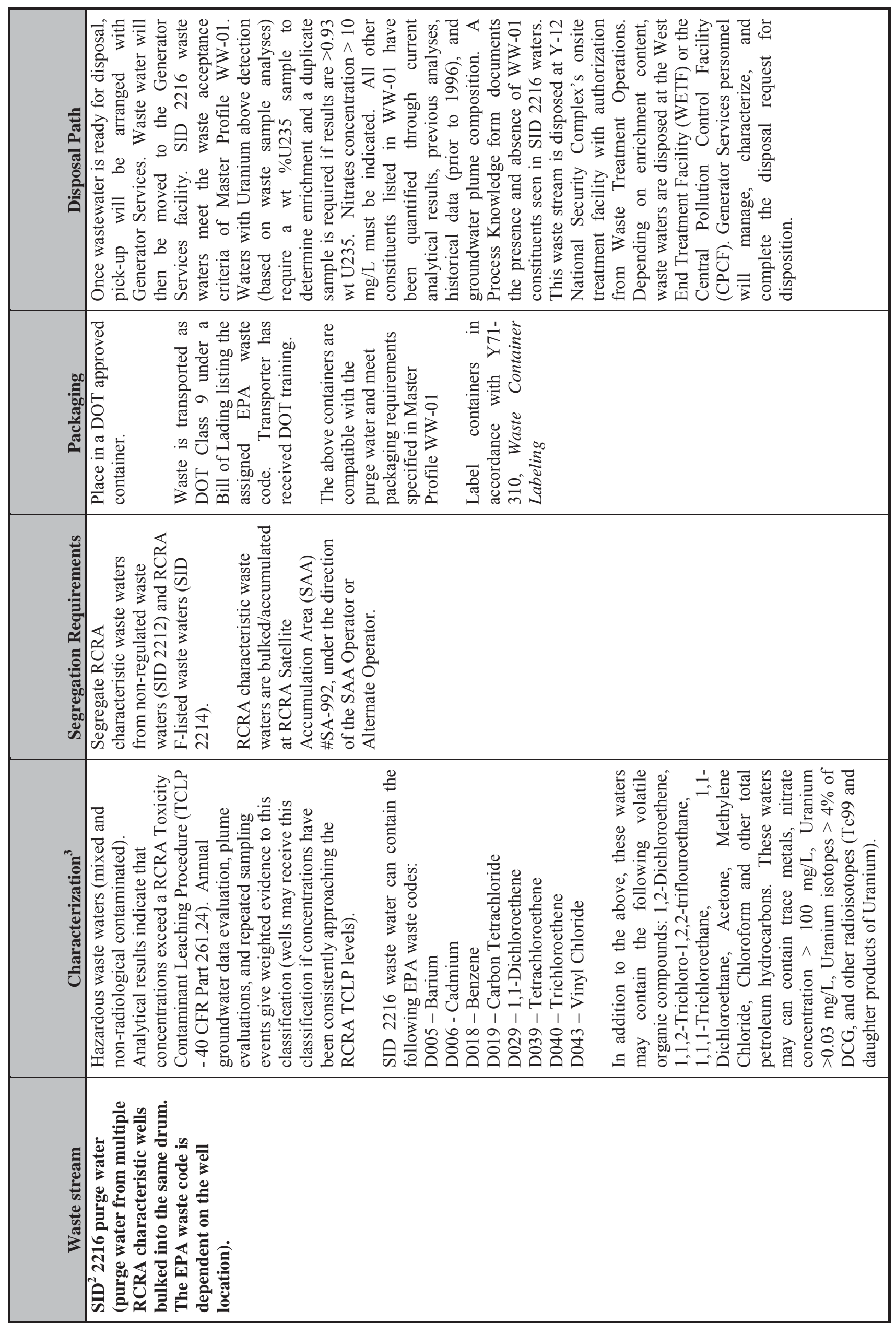

F1.1-7 


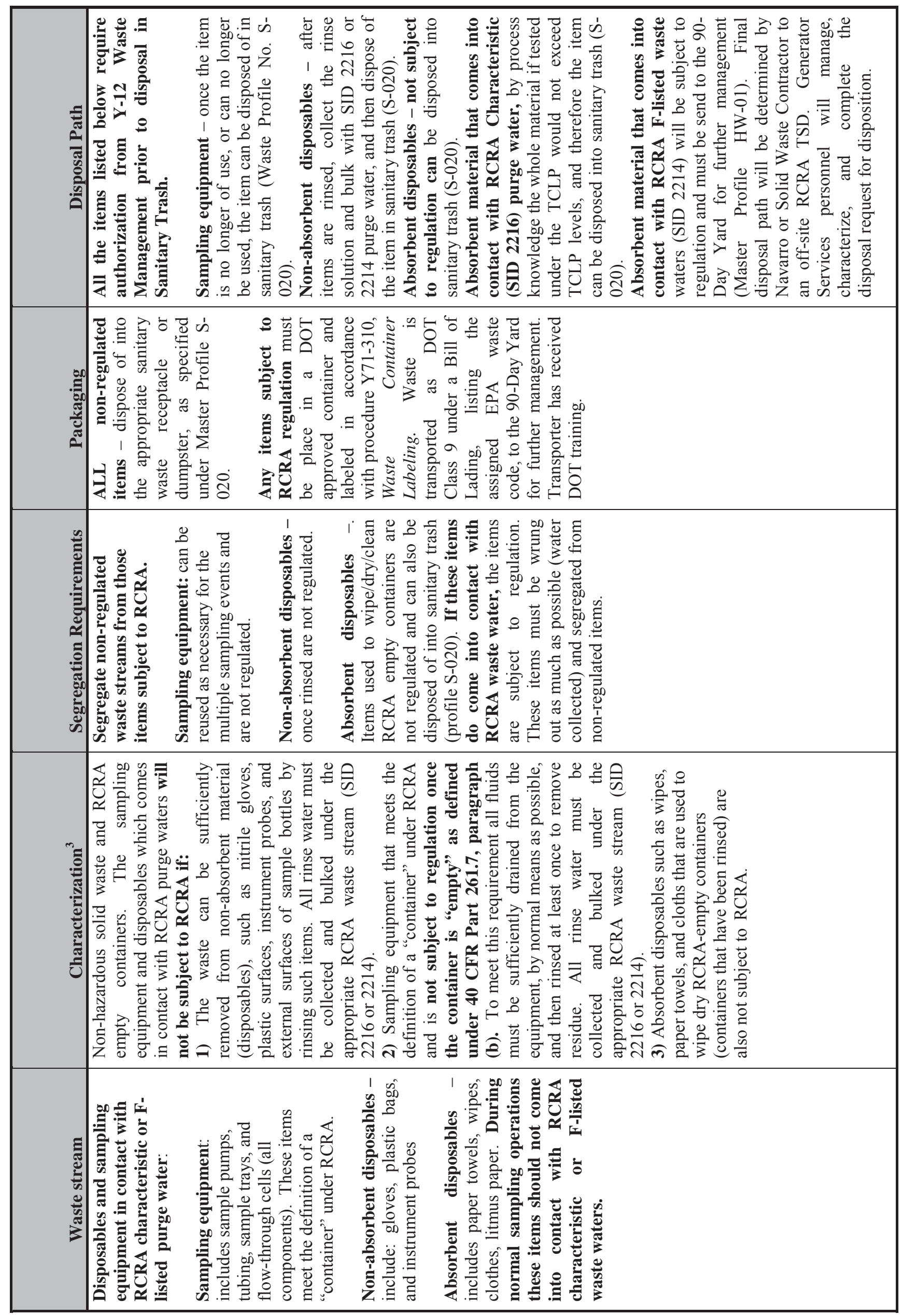




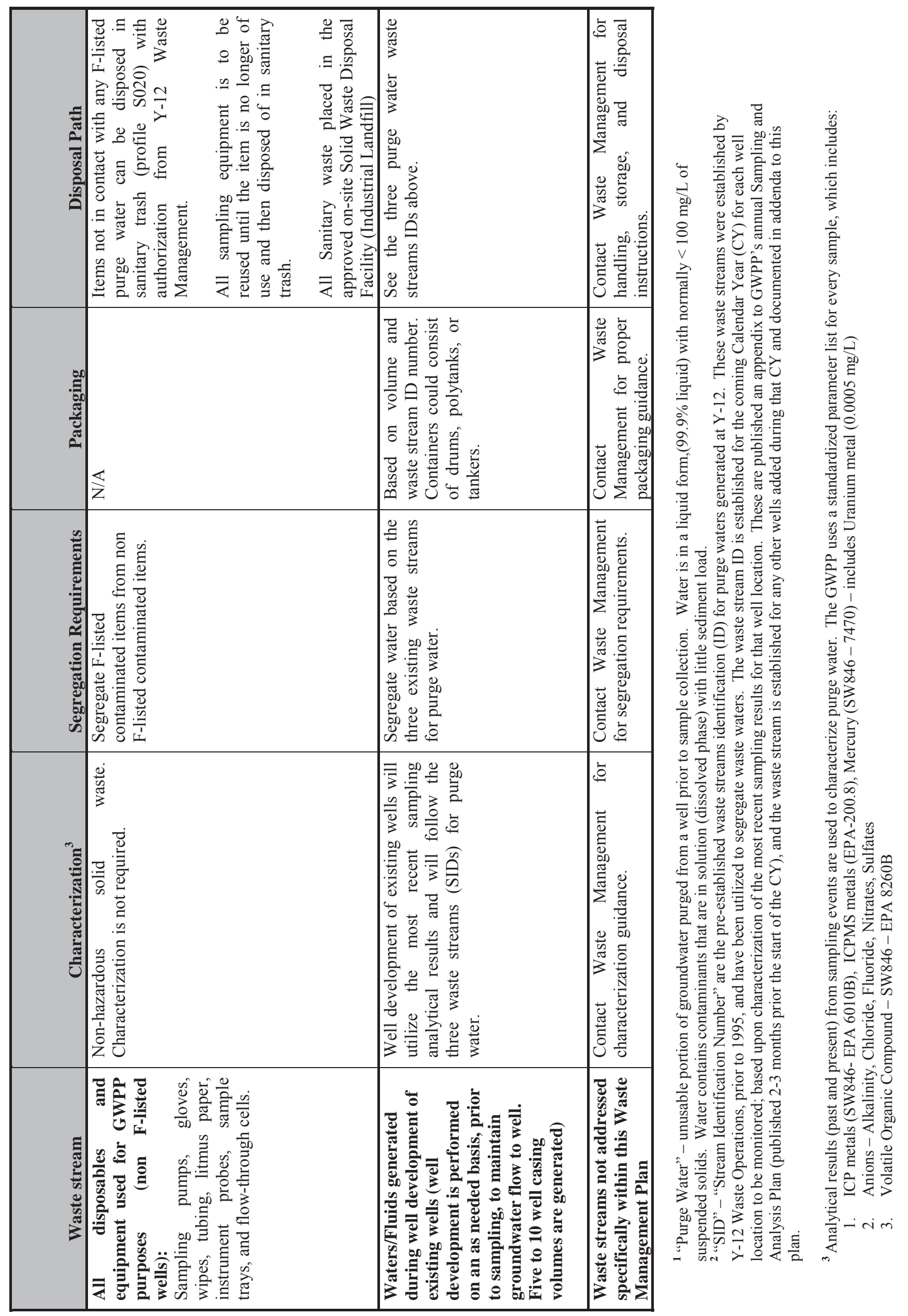

F1.1-9 


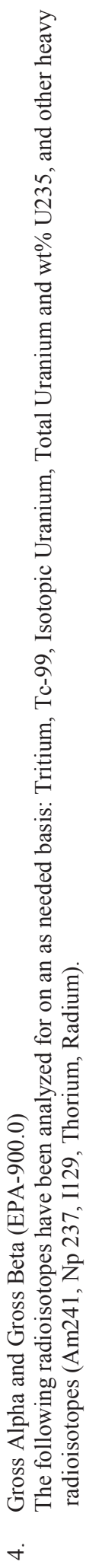

F1.1-10 
Appendix F.1

Y-12 GWPP PURGE WATER MANAGEMENT

Example of Waste Identification Tag (UCN 2114B)

SID 2212 purge water

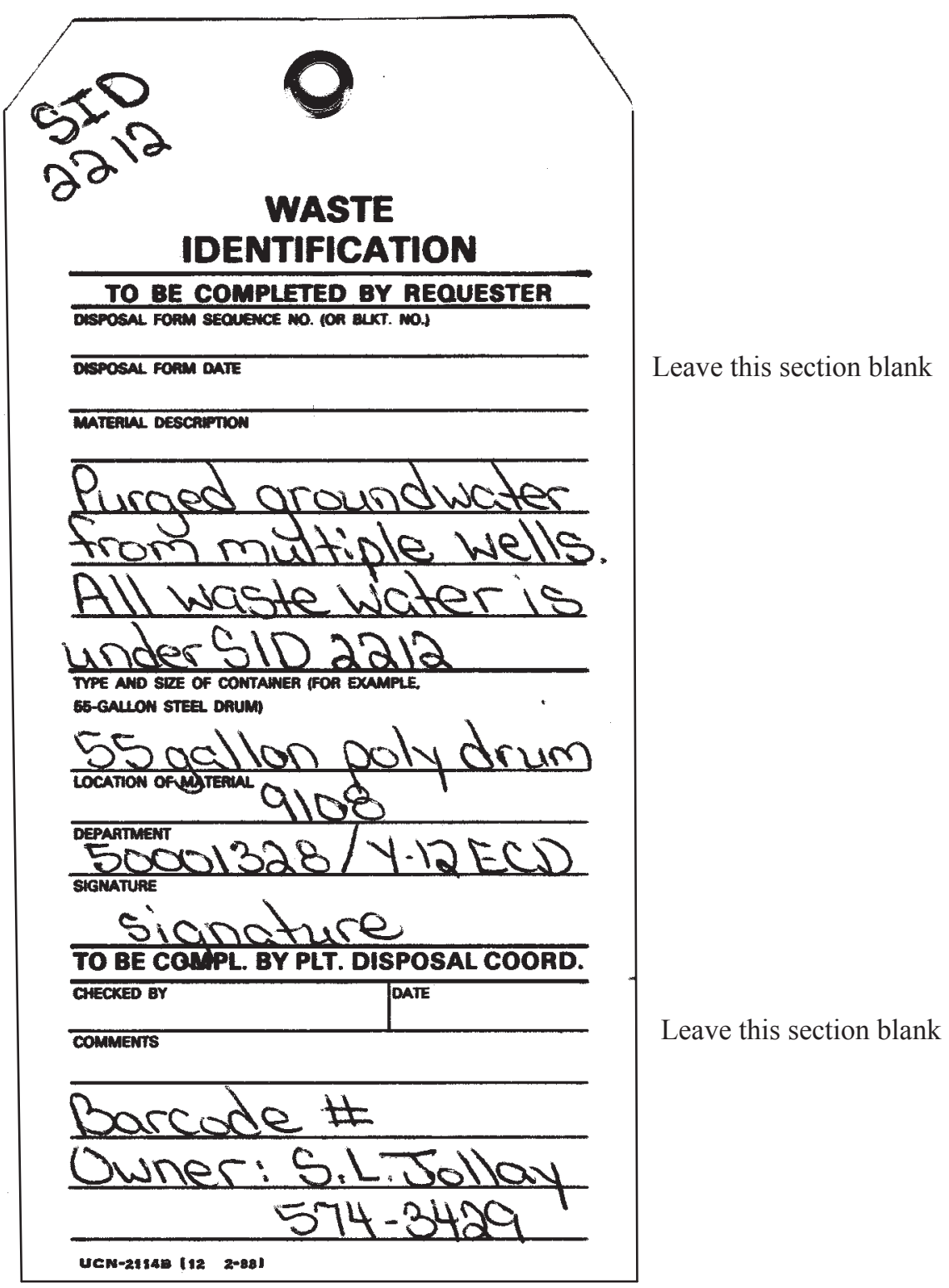




\section{Appendix F.1}

Y-12 GWPP PURGE WATER MANAGEMENT

Example of Waste Identification Tag (UCN 2114A)

SID 2214 purge water

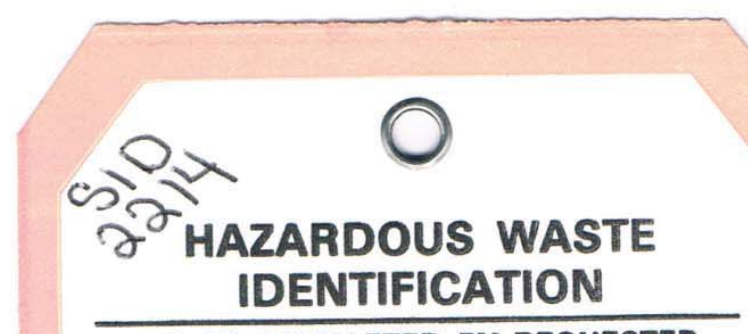

TO BE COMPLETED BY REQUESTER

DISPOSAL FORM SEQUENCE NO. (OR BLKT. NO.)

DISPOSAL FORM DATE

Leave this section blank

ACCUMULATION START DATE

MATERIAL DESCRIPTION

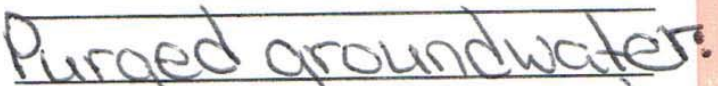

US EPA waste code: FO39

TYPE AND SIZE OF CONTAINER (FOR EXAMPLE,

55-GALLON STEEL DRUM)

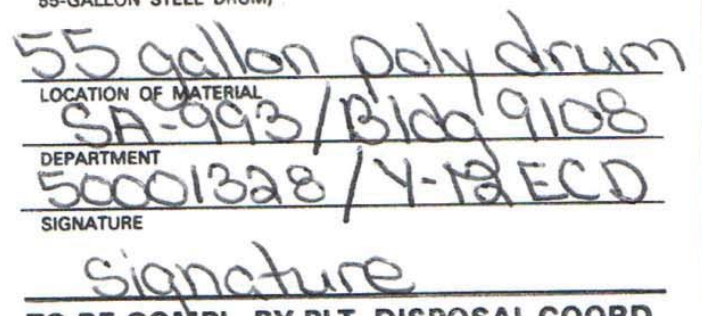

TO BE COMPL. BY PLT. DISPOSAL COORD. CHECKED BY

COMMENTS

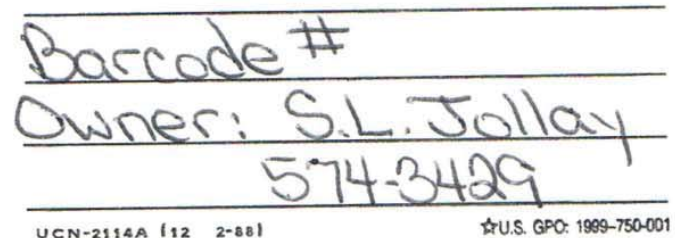

Leave this section blank 


\section{Appendix F.1}

Y-12 GWPP PURGE WATER MANAGEMENT

Example of Waste Identification Tag (UCN 2114A)

SID 2216 purge water

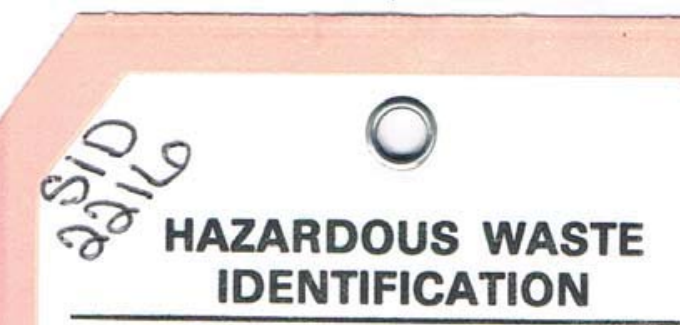

TO BE COMPLETED BY REQUESTER

DISPOSAL FORM SEQUENCE NO. (OR BLKT. NO.)

DISPOSAL FORM DATE

Leave this section blank

ACCUMULATION START DATE

MATERIAL DESCRIPTION

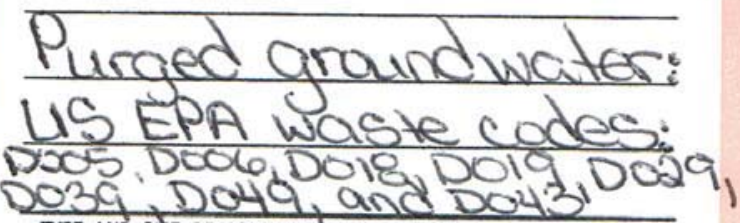
TYPE AND SILE OF CONTAINER (FOR EXAMPLE. 55-GALLON STEEL. DRUM)

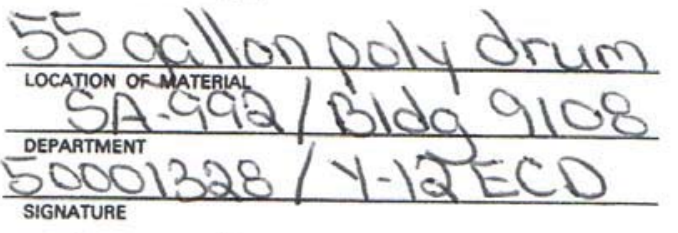
Sianature TO BE GOMPL. BY PLT. DISPOSAL COORD. CHECKED BY

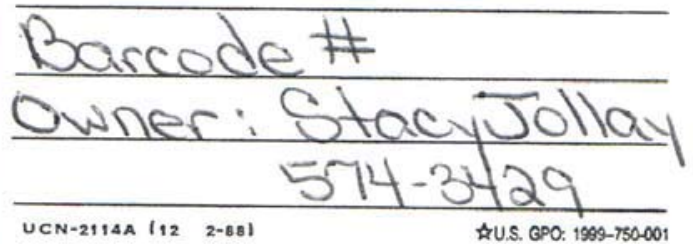


APPENDIX F.2

WASTEWATER STREAM IDENTIFICATION

FOR PURGED GROUNDWATER 
Table F.2. Waste stream identification and RCRA waste code for groundwater purged from wells to be sampled during CY 2014

\begin{tabular}{|c|c|c|c|c|c|c|c|}
\hline \multirow{2}{*}{ Regime } & \multirow{2}{*}{ Location } & \multicolumn{4}{|c|}{ CY 2014 Sampling Qtr. } & \multirow{2}{*}{$\begin{array}{c}\text { Waste Stream } \\
\text { Identification (SID \#) }\end{array}$} & \multirow{2}{*}{ RCRA Waste Code } \\
\hline & & Q1 & Q2 & Q3 & Q4 & & \\
\hline \multirow[t]{40}{*}{ Bear Creek } & GW-006 & & & $\mathrm{Y}$ & & Not Contained & \\
\hline & GW-014 & & & $\mathrm{Y}$ & & 2214 & F039 \\
\hline & GW-065 & & & $\mathrm{Y}$ & & 2212 & \\
\hline & GW-068 & & & $\overline{\mathrm{Y}}$ & & 2214 & F039 \\
\hline & GW-082 & $\mathrm{Y}$ & & & & 2214 & F039 \\
\hline & GW-085 & $\mathrm{Y}$ & & & & 2212 & . \\
\hline & GW-098 & & & $\mathrm{Y}$ & & 2212 & . \\
\hline & GW-100 & & & $\mathrm{Y}$ & & 2212 & . \\
\hline & GW-101 & & & $\overline{\mathrm{Y}}$ & & 2212 & . \\
\hline & GW-225 & & & $\mathrm{Y}$ & & 2212 & . \\
\hline & GW-229 & & & $\mathrm{Y}$ & & 2212 & . \\
\hline & GW-246 & $\overline{\mathrm{Y}}$ & & & & 2212 & . \\
\hline & GW-289 & $\mathrm{Y}$ & & & & 2214 & F039 \\
\hline & GW-307 & & & $\mathrm{Y}$ & & 2212 & . \\
\hline & GW-310 & & & $\mathrm{Y}$ & & 2212 & . \\
\hline & GW-312 & & & $\overline{\mathrm{Y}}$ & & 2212 & . \\
\hline & GW-315 & & & $\mathrm{Y}$ & & 2212 & . \\
\hline & GW-365 & & & $\mathrm{Y}$ & & 2212 & . \\
\hline & GW-526 & & & $\mathrm{Y}$ & & 2212 & . \\
\hline & GW-537 & $\overline{\mathrm{Y}}$ & & & & 2212 & . \\
\hline & GW-601 & & & $\overline{\mathrm{Y}}$ & & 2212 &. \\
\hline & GW-615 & & & $\mathrm{Y}$ & & 2216 & D005 \\
\hline & GW-616 & $\bar{Y}$ & & & & 2212 & \\
\hline & GW-623 & & & $\overline{\mathrm{Y}}$ & & 2214 & F039 \\
\hline & GW-626 & $\mathrm{Y}$ & & & & 2214 & F039 \\
\hline & GW-627 & $\mathrm{Y}$ & & $\overline{\mathrm{Y}}$ & & 2214 & F039 \\
\hline & GW-653 & & & $\overline{\mathrm{Y}}$ & & 2214 & F039 \\
\hline & GW-703 & & & $\overline{\mathrm{Y}}$ & & 2212 &. \\
\hline & GW-724 & & & $\mathrm{Y}$ & & 2212 & . \\
\hline & GW-725 & & & $\mathrm{Y}$ & & 2212 &. \\
\hline & GW-726-23 & $\overline{\mathrm{Y}}$ & & & & 2214 & F039 \\
\hline & GW-726-20 & $\overline{\mathrm{Y}}$ & & & & 2214 & F039 \\
\hline & GW-726-16 & $\mathrm{Y}$ & & & & 2214 & F039 \\
\hline & GW-726-12 & $\bar{Y}$ & & & & 2214 & F039 \\
\hline & GW-726-09 & $\overline{\mathrm{Y}}$ & & & & 2214 & F039 \\
\hline & GW-726-06 & $\bar{Y}$ & & & & 2214 & F039 \\
\hline & GW-726-04 & $\overline{\mathrm{Y}}$ & & & & 2214 & F039 \\
\hline & GW-738 & & & $\bar{Y}$ & & 2212 &. \\
\hline & GW-740 & & & $\mathrm{Y}$ & & 2212 & . \\
\hline & GW-829 & $\mathrm{Y}$ & & & & 2212 & . \\
\hline \multirow[t]{4}{*}{ Chestnut Ridge } & GW-322 & & $\bar{Y}$ & & & 2212 &. \\
\hline & GW-608 & & $\overline{\mathrm{Y}}$ & & & Not Contained &. \\
\hline & GW-609 & & $\bar{Y}$ & & & Not Contained & . \\
\hline & GW-611 & & $\overline{\mathrm{Y}}$ & & & 2212 & . \\
\hline \multirow[t]{5}{*}{ East Fork } & $55-2 \mathrm{~B}$ & & & & $\mathrm{Y}$ & 2212 & . \\
\hline & $55-2 \mathrm{C}$ & & & & $\mathrm{Y}$ & 2212 & \\
\hline & $55-3 \mathrm{~A}$ & & $\overline{\mathrm{Y}}$ & & $\mathrm{Y}$ & 2216 & D039, D040 \\
\hline & $55-3 \mathrm{~B}$ & & $\mathrm{D}$ & & $\overline{\mathrm{Y}}$ & 2216 & D039, D040, D043 \\
\hline & $55-3 \mathrm{C}$ & & $\mathrm{Y}$ & & $\mathrm{Y}$ & 2216 & D039, D040, D043 \\
\hline
\end{tabular}


Table F.2. (continued)

\begin{tabular}{|c|c|c|c|c|c|c|c|}
\hline \multirow{2}{*}{ Regime } & \multirow{2}{*}{ Location } & \multicolumn{4}{|c|}{ CY 2014 Sampling Qtr. } & \multirow{2}{*}{$\begin{array}{c}\text { Waste Stream } \\
\text { Identification (SID \#) }\end{array}$} & \multirow{2}{*}{ RCRA Waste Code } \\
\hline & & Q1 & Q2 & Q3 & Q4 & & \\
\hline \multirow{48}{*}{$\begin{array}{l}\text { East Fork } \\
\text { (continued) }\end{array}$} & $56-1 \mathrm{~A}$ & & & & Y & Not Contained & . \\
\hline & $56-1 \mathrm{C}$ & & & & $\mathrm{Y}$ & Not Contained & . \\
\hline & $56-2 \mathrm{~A}$ & & & & $\mathrm{Y}$ & 2212 & - \\
\hline & $56-2 \mathrm{~B}$ & & & & $\mathrm{Y}$ & 2216 & D039 \\
\hline & $56-2 \mathrm{C}$ & & & & $\mathrm{Y}$ & 2212 & . \\
\hline & $56-3 \mathrm{~A}$ & & & & $\mathrm{Y}$ & 2212 & . \\
\hline & $56-3 \mathrm{~B}$ & & & & $\mathrm{D}$ & 2212 & . \\
\hline & $56-3 \mathrm{C}$ & & & & $\overline{\mathrm{Y}}$ & 2212 & . \\
\hline & $56-4 \mathrm{~A}$ & & & & $\overline{\mathrm{Y}}$ & 2212 & . \\
\hline & $56-6 \mathrm{~A}$ & & & & $\mathrm{Y}$ & Not Contained & . \\
\hline & $60-1 \mathrm{~A}$ & & & & $\mathrm{Y}$ & Not Contained & . \\
\hline & GW-106 & & $\overline{\mathrm{Y}}$ & & & 2212 & . \\
\hline & GW-109 & & $\overline{\mathrm{Y}}$ & & & 2216 & D039 \\
\hline & GW-148 & & $\mathrm{Y}$ & & & 2212 & . \\
\hline & GW-153 & $\overline{\mathrm{Y}}$ & & & & 2212 & . \\
\hline & GW-204 & & & $\overline{\mathrm{Y}}$ & & 2212 & . \\
\hline & GW-220 & $\overline{\mathrm{Y}}$ & & $\mathrm{D}$ & & 2216 & D019 \\
\hline & GW-222 & & & & & 2212 & . \\
\hline & GW-240 & $\mathrm{D}$ & & & & 2212 & . \\
\hline & GW-251 & & & $\overline{\mathrm{Y}}$ & & 2212 & . \\
\hline & GW-265 & & & & $\bar{Y}$ & 2212 & . \\
\hline & GW-268 & & & & $\overline{\mathrm{Y}}$ & Not Contained & . \\
\hline & GW-269 & & & & $\mathrm{Y}$ & 2212 & . \\
\hline & GW-270 & & & & $\overline{\mathrm{Y}}$ & 2212 & . \\
\hline & GW-274 & & & & $\mathrm{D}$ & 2216 & D039 \\
\hline & GW-275 & & & & NP & 2216 & D005 \\
\hline & GW-332 & & & $\mathrm{Y}$ & & 2216 & D039 \\
\hline & GW-337 & & & $\mathrm{Y}$ & & 2212 & . \\
\hline & GW-381 & $\mathrm{Y}$ & & & & 2216 & D019 \\
\hline & GW-383 & $\mathrm{Y}$ & & & & 2212 & . \\
\hline & GW-619 & & $\mathrm{Y}$ & & & 2212 & . \\
\hline & GW-620 & & $\mathrm{Y}$ & & & Not Contained & . \\
\hline & GW-633 & & & $\overline{\mathrm{Y}}$ & & 2216 & D018 \\
\hline & GW-656 & $\mathrm{D}$ & & & & 2216 & D040 \\
\hline & GW-686 & & & $\mathrm{D}$ & & 2212 & . \\
\hline & GW-690 & & & $\mathrm{Y}$ & & 2212 &. \\
\hline & GW-691 & $\mathrm{Y}$ & & $\bar{Y}$ & & 2216 & D039 \\
\hline & GW-692 & & & $\mathrm{Y}$ & & 2212 & 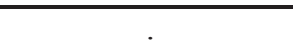 \\
\hline & GW-698 & $\overline{\mathrm{Y}}$ & & $\overline{\mathrm{Y}}$ & & 2216 & D040 \\
\hline & GW-700 & $\mathrm{Y}$ & & & & 2212 &. \\
\hline & GW-722-33 & $\mathrm{Y}$ & & & & Not Contained & . \\
\hline & GW-722-22 & $\mathrm{Y}$ & & & & 2212 & . \\
\hline & GW-722-14 & $\mathrm{Y}$ & & & & 2212 & . \\
\hline & GW-722-17 & $\mathrm{Y}$ & & & & 2212 & . \\
\hline & GW-722-20 & $\mathrm{Y}$ & & & & 2212 & . \\
\hline & GW-744 & $\mathrm{Y}$ & & & & Not Contained & . \\
\hline & GW-747 & $\mathrm{Y}$ & & & & Not Contained & . \\
\hline & GW-763 & $\mathrm{Y}$ & & & & 2212 & \\
\hline
\end{tabular}


Table F.2. (continued)

\begin{tabular}{|c|c|c|c|c|c|c|c|}
\hline \multirow{2}{*}{ Regime } & \multirow{2}{*}{ Location } & \multicolumn{4}{|c|}{ CY 2014 Sampling Qtr. } & \multirow{2}{*}{$\begin{array}{c}\text { Waste Stream } \\
\text { Identification (SID \#) }\end{array}$} & \multirow{2}{*}{ RCRA Waste Code } \\
\hline & & Q1 & Q2 & Q3 & Q4 & & \\
\hline \multirow{21}{*}{$\begin{array}{c}\text { East Fork } \\
\text { (continued) }\end{array}$} & GW-769 & & $\mathrm{Y}$ & & $\mathrm{Y}$ & 2212 & . \\
\hline & GW-770 & & $\overline{\mathrm{D}}$ & & & 2212 & . \\
\hline & GW-781 & $\bar{Y}$ & & & & Not Contained & . \\
\hline & GW-782 & $\mathrm{Y}$ & & & & 2212 & . \\
\hline & GW-783 & $\mathrm{Y}$ & & & & 2212 & . \\
\hline & GW-791 & $\mathrm{Y}$ & & & & 2212 & . \\
\hline & GW-792 & $\mathrm{Y}$ & & & & 2212 & . \\
\hline & GW-816 & $\mathrm{Y}$ & & & & Not Contained &. \\
\hline & GW-820 & & $\bar{Y}$ & & & 2216 & D039, D040 \\
\hline & GW-928 & & $\bar{Y}$ & & & Not Contained &. \\
\hline & GW-929 & & $\bar{Y}$ & & & Not Contained & . \\
\hline & GW-930 & & $\bar{Y}$ & & & Not Contained & . \\
\hline & GW-931 & & $\overline{\mathrm{Y}}$ & & & Not Contained & . \\
\hline & GW-934-12 & & & $\bar{D}$ & & 2216 & D019 \\
\hline & GW-934-11 & & & $\bar{Y}$ & & 2216 & D019 \\
\hline & GW-934-09 & & & $\overline{\mathrm{Y}}$ & & 2216 & D019 \\
\hline & GW-934-07 & & & $\mathrm{Y}$ & & 2216 & D019 \\
\hline & GW-934-05 & & & $\bar{Y}$ & & 2216 & D019 \\
\hline & GW-934-04 & & & $\overline{\mathrm{Y}}$ & & 2216 & D019 \\
\hline & GW-934-02 & & & $\overline{\mathrm{Y}}$ & & 2216 & D019 \\
\hline & GW-934-01 & & & $\bar{Y}$ & & 2216 & D019, D039 \\
\hline
\end{tabular}




\section{DISTRIBUTION}

\section{U.S. DEPARTMENT OF ENERGY}

J. P. Donnelly

PROGRAMS \& QUALITY

D. R. McDaniel

ENVIRONMENTAL COMPLIANCE DEPARTMENT

V. J. Brumback

S. M. Field

C. C. Hill

S. B. Jones

E. R. Schultz

S. E. McNamara

L. O. Vaughan

J. T. Williams

D. P. McDonald

\section{ANALYTICAL CHEMISTRY}

ORGANIZATION

L. K. Rawlins

\section{INFORMATION TECHNOLOGY}

S. W. King

9114DMC-01971865.6550-RC

Y-12 Records Services (Electronic copy- OSTI)

YDCC - RC
TENNESSEE DEPARTMENT OF ENVIRONMENT AND CONSERVATION

DOE OVERSIGHT

J. E. Sebastian

\section{$\underline{\text { ELVADO ENVIRONMENTAL LLC }}$}

T. R. Harrison

\section{RESTORATION SERVICES, INC}

T. S. Bunch

H. K. Haase

R. H. Ketelle

S. M. Selecman

L. M. Sims

File-EMEF-DMC

UT-BATTELLE, LLC

D. B. Watson 